\title{
High energy gamma rays from the Vela pulsar: long-term variability and energy distribution
}

\author{
I.A. Grenier ${ }^{1}$, W. Hermsen ${ }^{2}$, and J. Clear $^{3}$ \\ ${ }^{1}$ Service d'Astrophysique, Centre d'Etudes Nucléaires de Saclay, F-91191 Gif-sur-Yvette Cedex, France \\ ${ }^{2}$ Laboratory for Space Research Leiden, Leiden, The Netherlands \\ ${ }^{3}$ Space Science Department of ESA, ESTEC, Noordwijk, The Netherlands
}

Received December 7, 1987; accepted March 11, 1988

\begin{abstract}
Summary. New results on the temporal and spectral characteristics of the high energy ( $50 \mathrm{MeV}$ to $5 \mathrm{GeV}$ ) gamma ray emission from the Vela pulsar are presented. A new sensitive analysis method using maximum likelihood techniques has been applied to the final COS-B dataset. The whole pulsed flux is found to exhibit long term variability. The energy spectrum of this total emission cannot be described by a single power law and the data show strong evidence for a spectral break at approximately $300 \mathrm{MeV}$. Five discrete emission regions within the pulsar lightcurve have been identified, with the spectral characteristics and long term behaviour being different. These results support the idea that various source regions simultaneously exist in the pulsar magnetosphere and that the physical processes generating the gamma rays in these sites differ with the location (in altitude and latitude).
\end{abstract}

Key words: PSR 0833-45 - Vela - pulsars - gamma rays COS-B-pulsar glitch

\section{Introduction}

The study of the high energy gamma ray emission from celestial objects provides an excellent method in helping our understanding of the physical processes which occur within such sources. The various possible emission mechanisms are well understood, with the gamma ray emission being closely related to the central power house of the source. A detailed knowledge of the emission characteristics therefore leads to such important properties as the nature of the process, particle densities and spectra, and the strength of the magnetic field. The Vela pulsar (PSR 0833-45) is a most unusual galactic object and is one of the fastest pulsars known. First discovered by its pulsed radio emission (Large et al., 1968), it was at the time of its detection the weakest object ever observed in the visible light, while being the brightest source in the gamma ray sky. The pulsar luminosity reaches a maximum in the gamma ray domain but it has not been detected at X-ray energies. Furthermore, its lightcurve structure varies considerably with the energy range. Clearly, the study of the high energy gamma ray emission from the pulsar provides a useful method in

Send offprint requests to: I.A. Grenier understanding the complex energetic processes which occur in its magnetosphere.

Figure 1 shows the pulsar lightcurve at radio, optical and gamma ray energies. The radio signal (Buccheri et al., 1978) shows a single pulse arriving about a third of a period before the common mid-point of the other lightcurves. Its profile depends on the frequency and on the intensity of the emission, and could be the result of four components generated at different altitudes just above the polar cap (Krishnamohan and Downs, 1983). The pulsar was observed at optical wavelengths by Wallace et al. (1977) and Peterson et al. (1978) as a 24th magnitude star with two broad optical pulses, separated by 0.25 in phase, on top of a background light level which accounts for half the luminosity of the pulsar. Manchester et al. (1980) find evidence for a slight modulation of the baseline seen in the optical lightcurve. The pulsar has long been recognized as the X-ray source, 3U 0833-45, over a wide range of energies (Kellogg et al., 1973; Culhane et al., 1974; Pravdo et al., 1976 and references therein). Accurate observations by the Einstein satellite recently resolved the source into a point-source coincident with the pulsar position and embedded in a tiny $\left(4^{\prime}\right)$ nebula of diffuse emission (Harnden et al., 1985), but no pulsed emission was observed. Results from other experiments (Knight et al., 1982 and references therein) report only upper limits to the pulsed emission. However, two positive detections have been reported in the past (Harnden et al., 1972, and Harnden and Gorenstein, 1973) but the results have not been confirmed. Contrarily, the reported fluxes are not consistent with these upper limits and the derived phase distribution and pulsar period respectively, are inconsistant with the other reports. The first detection of pulsed gamma ray emission from the Vela pulsar was reported by Albats et al. (1974) in the $10-30 \mathrm{MeV}$ region. The lightcurve showed a single peak almost coincident in phase with the radio peak, however this result has never been confirmed. Tümer et al. (1984) at 0.3-30 MeV, Thompson et al. (1977) above $35 \mathrm{MeV}$ and Bennett et al. (1977) at $50 \mathrm{MeV}-5 \mathrm{GeV}$ all report a similar lightcurve structure which is characterised by two sharp peaks separated by 0.42 in phase and bracketing the optical pulses. At $\mathrm{TeV}$ energies pulsed emission has been reported from Vela at a low statistical significance, with the position and number of the peaks in the lightcurve varying (Bhat et al., 1980; Gupta et al., 1982; and Bhat et al., 1987). The energy spectrum of the pulsar still remains uncertain. An extrapolation to X-ray energies of the COS-B results of Kanbach et al. (1980) lies several orders of magnitude above the reported upper limits, while at 


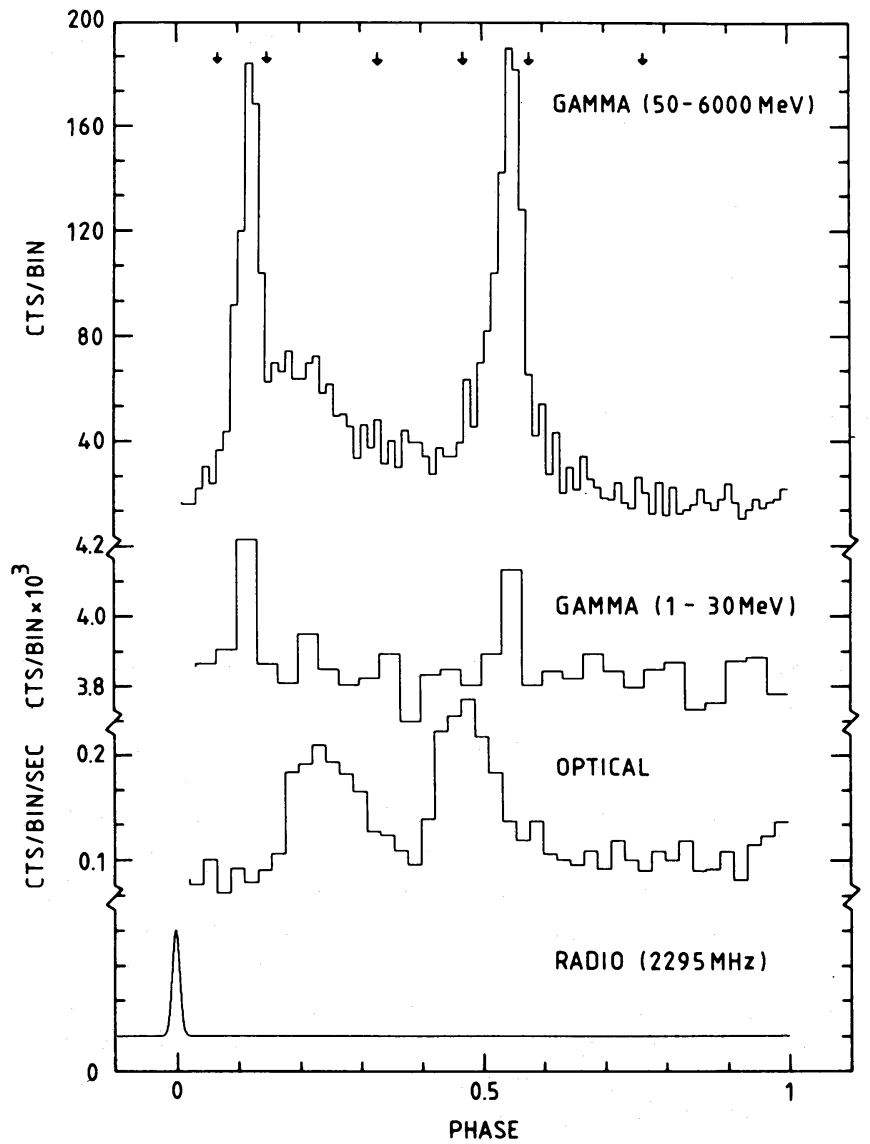

Fig. 1. The lightcurve from PSR 0833-45 at radio (Buccheri et al., 1978), optical (Manchester et al., 1980), soft energy gamma rays (Tümer et al., 1984) and high energy gamma rays (this work). The arrows indicate the position of the phase boundaries which are used in this analysis

$\mathrm{TeV}$ energies the flux values reported by Bhat et al. (1980) clearly require a break in the spectrum. Earlier results from COS-B data by Bennett et al. (1977) and Kanbach et al. (1980) indicate a possible break in the spectrum at a few hundred $\mathrm{MeV}$, but the level of significance of these results is low. Finally, Caraveo et al. (1987) have recently reported a possible detection of linear polarisation in the high energy gamma ray emission using COS-B data. If confirmed, this result will strongly support an electromagnetic production mechanism of the gamma rays from the pulsar.

This article presents the latest results on the Vela pulsar in the $50 \mathrm{MeV}$ to $5 \mathrm{GeV}$ energy range. The data have been collected using the COS-B satellite which observed the Vela region during 10 observation periods, that is for more than 300 days and a total exposure of $1.4810^{8} \mathrm{~cm}^{2} \mathrm{~s}$. Whereas most of this dataset has been used previously to study the pulsar timing parameters and lightcurve structure (Wills et al., 1981), only a small fraction has been used to examine its spectral characteristics (Bennett et al., 1977; Lichti et al., 1980; Kanbach et al., 1980), and no previous study of the long term behaviour of the pulsar luminosity and spectral properties has been reported. Initially, the details of the observations and the results of a preliminary study using the traditional analysis method are presented. Subsequently, a detailed description is given of a sensitive maximum likelihood analysis method developed to study the spectral characteristics of the pulsed emission and its long term behaviour. Finally, the results of this analysis are presented and a discussion is given of how this detailed description of the Vela pulsar's behaviour at high energies may constrain the theoretical modelling of the source.

\section{Observations}

The gamma ray satellite COS-B operated in the $50-5000 \mathrm{MeV}$ range. Details of the spark chamber experiment are given by Scarsi et al. (1977). For this analysis, data from the 'final COS-B database' have been used (Mayer-Hasselwander et al., 1985). The database contains information on each selected photon, together with the results of the pre-flight and in-flight calibration data on the instrumental Point-Spread-Function (PSF), on the energy dispersion probability (both as a function of energy and source aspect angle), and on the relative instrumental sensitivity for each observation. The latter shows a generally decreasing trend during the COS-B lifetime due to the degradation of the spark chamber efficiency. Its evolution was estimated from the study of overlapping observations along the galactic plane (Strong et al., 1987a), so that the sensitivity during an observation is known with an accuracy of $10 \%$ (or better when the observation refers to a repeatedly surveyed region). During its 6.7 years of almost continuous operation a total of 65 individual observations were made, each lasting typically 30 to 40 days. The Vela pulsar was in the COS-B field of view during the 10 observations in Table 1.

\section{Preliminary results}

For temporal analysis, the data have been folded with the pulsar timing parameters applicable to the particular epoch of the observation. These were derived from contemporary radio measurements for periods 2, 3, 12, 21 and 45, and for the remaining observations parameter scanning using the COS-B data itself was performed. In order to generate lightcurves, events have been selected from within an energy dependent cone of half angle $\theta_{\max }$ $=12.5 \mathrm{E}_{\mathrm{GeV}}^{-0.16}$ degrees about the pulsar position. This expression maximizes the signal to noise ratio within the lightcurve for the energy dependent PSF (Buccheri et al., 1983). Figure 1 shows the resultant lightcurve obtained by summing the data over all observations and compares it to the pulsed emission recorded at other wavelengths. Also indicated in Fig. 1 are the boundaries which are used in this analysis to study the background and phase dependence of the emission. The boundaries are specified in Table 2.

The entire data set was initially analysed using the 'traditional' saturation method described in detail by Bennett et al. (1977). It exploits the information given by the background region of the lightcurve to determine the number of pulsed photons for a particular energy interval and phase region. Flux values may then be calculated directly from the useful observing time and effective sensitive area for an assumed source spectral index. The saturation method provides a well established quick-look analysis method which can handle data up to large aspect angles, and also facilitates verification of results derived using the maximum likelihood method which is described in detail in Sect. 4.

To study the long-term behaviour of the pulsar, the total pulsed flux in the energy interval $50-5000 \mathrm{MeV}$ was determined for each observation period for an assumed source spectral index of -1.89 (the index determined by Kanbach et al., 1980, using 
Table 1. Characteristics of each Vela observation

\begin{tabular}{|c|c|c|c|c|c|}
\hline $\begin{array}{l}\text { Observation } \\
\text { period }\end{array}$ & $\begin{array}{l}\text { Pointing } \\
\text { direction }(l, b) \\
\text { (degrees) }\end{array}$ & $\begin{array}{l}\text { Vela } \\
\text { aspect } \\
\text { angle }\left(^{\circ}\right)\end{array}$ & $\begin{array}{l}\text { Start, end } \\
\text { dates } \\
(\mathrm{d} / \mathrm{m} / \mathrm{y})\end{array}$ & $\begin{array}{l}\text { Relative } \\
\text { efficiency }\end{array}$ & $\begin{array}{l}\text { Vela exposure } \\
50 \mathrm{MeV}-5 \mathrm{GeV} \\
\left(10^{7} \mathrm{~cm}^{2} \mathrm{~s}\right)\end{array}$ \\
\hline 2 & $264,-3$ & 1 & $\begin{array}{l}20 / 10 / 75 \\
08 / 11 / 75\end{array}$ & 1.02 & 2.44 \\
\hline 3 & $263,+4$ & 6 & $\begin{array}{l}08 / 11 / 75 \\
28 / 11 / 75\end{array}$ & 1.03 & 2.06 \\
\hline 5 & 292,0 & 29 & $\begin{array}{l}24 / 12 / 75 \\
23 / 01 / 76\end{array}$ & 0.94 & 0.25 \\
\hline 12 & $263,+3$ & 6 & $\begin{array}{l}24 / 07 / 76 \\
24 / 08 / 76\end{array}$ & 0.72 & 2.61 \\
\hline 20 & $260,+18$ & 21 & $\begin{array}{l}14 / 04 / 77 \\
02 / 05 / 77\end{array}$ & 0.66 & 0.56 \\
\hline 21 & $243,-2$ & 20 & $\begin{array}{l}02 / 05 / 77 \\
08 / 06 / 77\end{array}$ & 0.68 & 1.26 \\
\hline 40 & $243,-3$ & 20 & $\begin{array}{l}03 / 04 / 79 \\
09 / 05 / 79\end{array}$ & 0.84 & 0.82 \\
\hline 42 & 278,0 & 17 & $\begin{array}{l}20 / 06 / 79 \\
27 / 07 / 79\end{array}$ & 0.72 & 0.73 \\
\hline 45 & $263,+4$ & 7 & $\begin{array}{l}10 / 10 / 79 \\
15 / 11 / 79\end{array}$ & 0.73 & 2.25 \\
\hline 59 & $269,-10$ & 9 & $\begin{array}{l}09 / 04 / 81 \\
03 / 06 / 81\end{array}$ & 0.53 & 1.80 \\
\hline
\end{tabular}

Table 2. Definition of the selected phase intervals of the Vela pulsar lightcurve

\begin{tabular}{ll}
\hline Region & Phase interval \\
\hline Peak 1 & $0.07-0.15$ \\
Interpulse-1 & $0.15-0.33$ \\
Interpulse-2 & $0.33-0.47$ \\
Peak 2 & $0.47-0.58$ \\
Trailer & $0.58-0.77$ \\
Background & $0.77-0.07$ \\
\hline
\end{tabular}

observation periods 2 and 3 only). Figure 2 shows the evolution of this flux from 1975 to 1981 . The error bars indicated in the figure are statistical only and do not account for uncertainties in the instrument response. The results show significant flux variability from the pulsar. Including the uncertainty in the instrument response $(10 \%)$ the chance probability of this effect lies well below $10^{-7}$.

To determine the time averaged spectral characteristics of the source, the pulsed flux was calculated using the data from all 10 observations and for 9 energy intervals (the effective sensitive area was derived assuming again a source spectral index of -1.89 ). Figure 3 shows the resultant spectrum. A single power law fit to the points yields a spectral index of -1.92 for the entire energy range. However, the probability that the data is well represented by such a spectrum is less than $10^{-3}$. By restricting the fit to energies above $300 \mathrm{MeV}$ a single power law fit of

$F_{\gamma}(E)=(2.62 \pm 0.13) 10^{-3} E_{\mathrm{MeV}}^{-2.15 \pm 0.07}$ photon $\mathrm{cm}^{-2} \mathrm{~s}^{-1} \mathrm{MeV}^{-1}$

has been found to well represent the data with a probability of 0.8 . Clearly, a second power law or an even more complicated model is required for energies between 50 and $300 \mathrm{MeV}$.

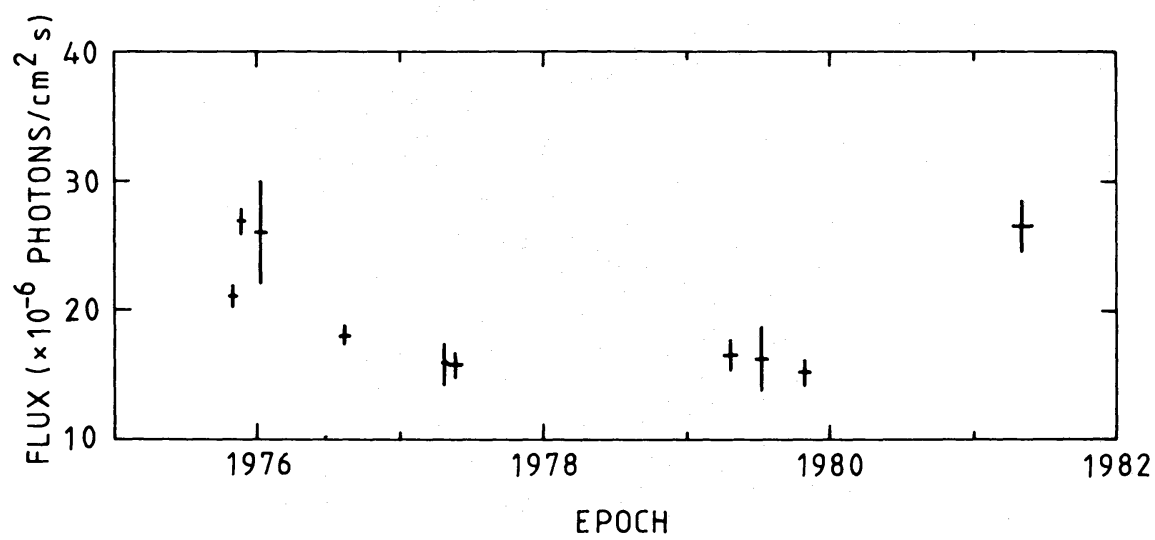

Fig. 2. Integrated gamma ray flux (50-5000 MeV) from the Vela pulsar at various epochs from 1975 to 1982 . The flux values are calculated using the saturation method and an assumed source spectral index of -1.89 


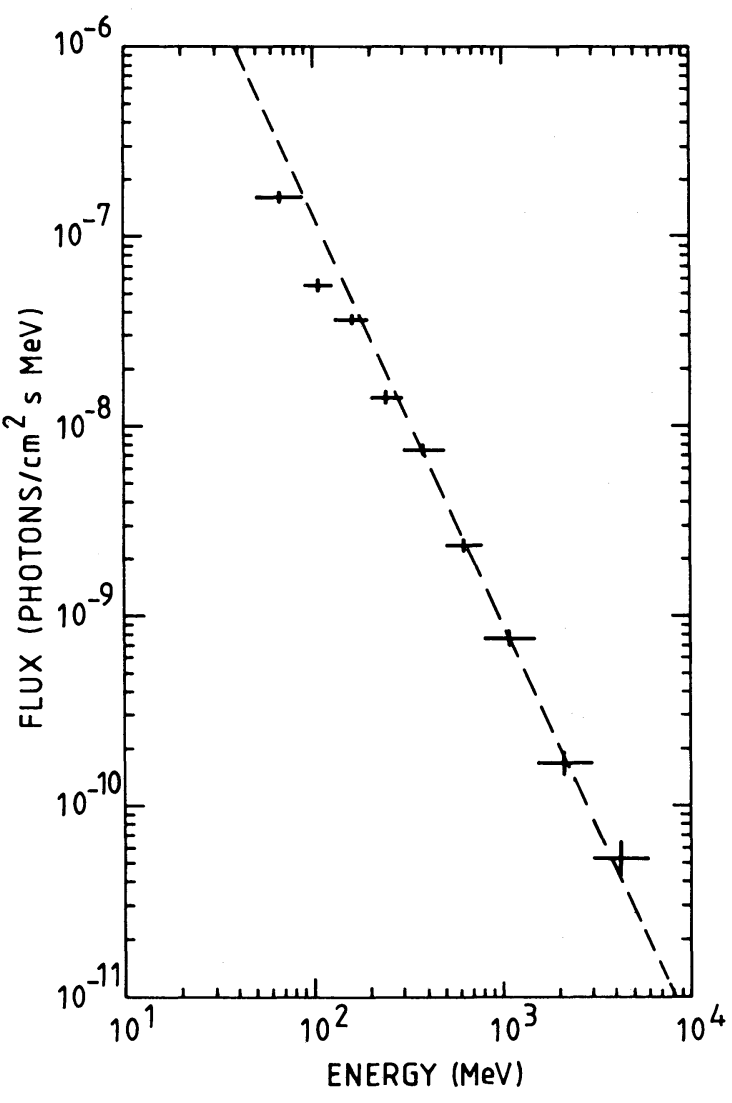

Fig. 3. Phase averaged differential gamma ray spectrum from the Vela pulsar using all COS-B observations. The dashed line represents the best fit to the spectrum at energies above $300 \mathrm{MeV}$

Finally, as part of the preliminary analysis, the background region of the lightcurve has been analysed for possible steady emission from the Vela region lying above the diffuse galactic emission. The analysis method has been described in detail by Clear et al. (1987). Table 3 shows the results for 4 energy intervals. The value $\alpha$ represents the $95 \%$ confidence limit to the ratio of the pulsed to unpulsed emission from Vela. For each energy range, no evidence exists within the statistical uncertainties for the detection of steady emission from Vela. Over the total energy range a $95 \%$ confidence level upper limit of $1.1510^{-6}$ photon $\mathrm{cm}^{-2} \mathrm{~s}^{-1}$ has been determined.

The results of the preliminary analysis show the Vela pulsar to exhibit states of high and low activity during the epoch of the observations. The energy spectrum cannot be described by a single power law and there is evidence for a spectral break at

Table 3. Upper limits to the ratio $(\alpha)$. of the unpulsed to pulsed emission for four energy ranges

\begin{tabular}{ll}
\hline $\begin{array}{l}\text { Energy range } \\
(\mathrm{MeV})\end{array}$ & $\begin{array}{l}\text { Unpulsed/pulsed } \\
(\alpha)\end{array}$ \\
\hline $50-100$ & 0.08 \\
$100-200$ & 0.09 \\
$200-500$ & 0.07 \\
$500-5000$ & 0.06 \\
\hline
\end{tabular}

approximately $300 \mathrm{MeV}$. In order to study the phase and spectral characteristics in greater detail a more sensitive analysis method is required. For this purpose a maximum likelihood analysis method has been developed, which simultaneously considers all COS-B information for each event, and which forms a complete model of the gamma ray emission from the Vela region. The details of this method are now described.

\section{The maximum likelihood method}

A maximum likelihood analysis has been applied to the data to estimate the flux and spectral properties of the source. The newly developed approach utilizes all COS-B information, that is the arrival time, energy and direction of each photon. It takes simultaneously into account the limited spatial and energy resolutions of the instrument, and their dependency on the inclination and energy of an event. Finally, it separates the pulsar emission from any background radiation by exploiting the pulsar lightcurve shape against the flatness of the background, and the source spatial structure (the COS-B point-spread-function) against the structure of the underlying diffuse emission originating in the interstellar medium and the isotropy of the instrumental noise. The statistical processing uses the maximum likelihood principle with a probability density function of detecting a photon at an energy $E_{m}$, position $l_{m}, b_{m}$ and phase $\phi_{m}$ (where $m$ is for 'measured' against 'true') expressed as:

$\frac{\int_{E} \iint_{l b} n(\phi, E, l, b) d E d \Omega \operatorname{CAL}\left(E_{m}, E, \alpha_{m l b}, \theta_{l b}\right)}{\int_{E_{m}} \iint_{l_{m b m}} \text { Numerator } d E_{m} d \Omega_{m}}$

The true celestial gamma ray distribution is described by $n(\phi, E, l$, b). CAL represents the COS-B convolution, given by the product of its effective sensitive area, point-spread-function and energy resolution. These parameters have been determined from prelaunch calibration data (Hermsen, 1980, Mayer-Hasselwander, 1985). $\theta_{l b}$ represents the true inclination angle with respect to the detector axis of the photons coming from a point source at $(l, b)$ and reconstructed at an angle $\alpha_{m l, b}$ away from this source. $\Omega$ denotes the solid angle. The likelihood value can then be computed from the product of the probabilities of finding the photons actually recorded by COS-B.

For the Vela gamma ray source, the general model to test may include up to four major components:

1. the pulsed emission from the pulsar: $F_{v p}(\phi) E^{-\gamma_{p}(\phi)} \delta\left(l-l_{v}\right.$, $\left.b-b_{v}\right)$;

2. a possible steady component from Vela: $F_{v s} E^{-\gamma_{s}} \delta\left(l-l_{v}\right.$, $\left.b-b_{v}\right)$

3. a constant and isotropic instrumental background: $B_{I} E^{-\gamma_{i}}$;

4. the steady diffuse galactic emission around the pulsar: $q N H(l, b) E^{-\gamma_{g}}$.

The latter term results from the interation of cosmic rays with interstellar matter. Its spatial structure can be traced by the $\mathrm{H}_{\mathrm{I}}$ and CO surveys (Dame et al., 1987; Strong et al., 1987b). To predict the galactic gamma ray distribution, $3^{\circ} \times 3^{\circ}$ interstellar maps showing $N H(l, b)$ within $30^{\circ}$ around Vela have been constructed assuming a value of $2.810^{20} \mathrm{~mol} \mathrm{~cm}^{-2} \mathrm{~K}^{-1} \mathrm{~km}^{-1} \mathrm{~s}$ for $\mathrm{NH}_{2} /$ WCO (Bloemen et al., 1986). For each observation the entire COS-B field of view has been covered.

Testing different models is performed by evaluating the likelihood expression for the parameters of the different components: $B_{I}, q, F_{v s}, F_{v p}(\phi)$, and the different spectral slopes: $\gamma_{i}, \gamma_{g}, \gamma_{s}$, 
$\gamma_{p}(\phi)$. The highest likelihood value gives the most probable model $n(\phi, E, l, b)$ that explains the data. As a boundary condition, the total number of photons from a selected model is assumed to equal the total number of photons detected within the entire COS-B field of view $\left(60^{\circ}\right)$ and energy range (50-5000 MeV). This number depends only on the phase and is not altered by COS-B. The good timing resolution of the instrument $(1 \mathrm{~ms})$ allows the phase of each event to be derived to an accuracy of 0.01 . Thus for the analysis, the measured phase may be taken as the actual phase of each event. This is an important condition as the spectral characteristics from adjacent phase intervals may therefore be analysed independently. Table 2 and Fig. 1 give the details of the six phase domains which have been selected for the analysis. The specific choice of these domains is based on the apparent structure in the gamma ray lightcurve following Kanbach et al. (1980), the possible physical relationship between the gamma ray structure in interpulse-1 and the first optical peak and on the requirement to maintain good statistics. The background region (phase $0.77-0.07$ ) has been analysed as part of the preliminary analysis to search for a possible steady emission from Vela above the diffuse galactic emission. The resultant upper limit for the total energy range (see Sect. 3) shows that such emission accounts for less than a few per-cent of the pulsed luminosity, and may thus be neglected from the model. Ignoring possible steady emission in the model implies that outside the pulsed phase range, the photons only come from the two background components of the model. As a first step, the likelihood analysis is performed for the events from the background phase domain to give the best emissivities and spectral indicies of the instrumental background and galactic emission $\left(B_{I}, \gamma_{i}, q, \gamma_{g}\right)$. These values are then fixed in the model and the analysis is applied to the photons from the other five phase intervals independently to derive the emissivities $F_{V P}(\phi)$ and spectral indicies $\gamma_{p}(\phi)$ of the pulsed emission within each selected phase. Changing the background set of parameters within the $1 \sigma$ level around their most likely value has been found not to affect the results of the pulsar analysis, at least well within the quoted errors.

This type of analysis is more precise and powerful than the traditional saturation method such that the total pulsed spectra as well as the spectra for the selected phase intervals can be determined for individual observations. However, for those observations with large source aspect angles $\left(>15^{\circ}\right)$, where the efficiency and the energy resolution of the instrument are poor, this likelihood analysis is limited by the available statistics and has not been performed. For the remaining observations $(2,3,12$, 45 and 59) a detailed analysis has been made for the 5 phase intervals. Within each phase interval the analysis has been performed for 3 energy intervals $(50-5000 \mathrm{MeV}, 50-300 \mathrm{MeV}$ and 300-5000 MeV). The division of the data into these energy intervals is based on the evidence for a break in the pulsed spectrum at approximately $300 \mathrm{MeV}$ found in the preliminary analysis. Due to computing time considerations the position of the spectral break has not been used as an additional free parameter within the model, however the results of the independent modelling of the spectrum above and below $300 \mathrm{MeV}$ strongly supports the choice of this value.

\section{Results}

The likelihood analysis has been performed for the 5 selected phase intervals, for the 5 best observation periods and for 3 energy ranges, giving a total of 75 spectra and flux values. The results have been summarized in Table 4 . The spectral slopes have been scanned over a wide range (typically 0 to 4 ) and the derived regular parabolic shapes of all the likelihood functions denote that the parameters have been well optimized. The quoted errors have been determined from the shapes of the likelihood ratio functions $\lambda$ (defined as the ratio between the likelihood maximized over the background parameters and the likehood maximized over all parameters). Since in the present case the quantity $-2 \ln (\lambda)$ has a $\chi^{2}$ distribution with one degree of freedom, $\gamma_{p}(\phi)$, the $68 \%$ confidence level corresponds to a decrease in $-2 \ln (\lambda)$ of 1.0. Probability estimates are given in Table 4 to evaluate the chance probability of a change in the spectral shape with phase against the assumption of a homogeneous pulsed spectrum, and to examine the time variability of the spectral slopes. Details of how the likelihood analysis permits a qualitative comparison between two models are given by Eadie et al. (1982). To study the long-term evolution of the fluxes, $\chi^{2}$ values are given. In the following discussion these results shall be considered in detail giving a coherent image of the behaviour of the Vela pulsar in the gamma ray domain.

Initially, consider the time averaged, phase averaged behaviour of the pulsar. The single power law which best describes the pulsed emission over the energy range $50-5000 \mathrm{MeV}$ has an index of $1.84 \pm 0.03$. This can be compared with the value of $1.89 \pm 0.06$ calculated by Kanbach et al. (1980) using periods 2 and 3 . The spectrum of the whole pulsed emission derived independently at energies above and below $300 \mathrm{MeV}$ and using all observations is:

$$
\begin{array}{r}
F(50 \leq E \leq 300)=(2.74 \pm 0.21) 10^{-4} E_{\mathrm{MeV}^{-1.72 \pm 0.07}} \\
\text { photon } \mathrm{cm}^{-2} \mathrm{~s}^{-1} \mathrm{MeV}^{-1} \\
F(300 \leq E \leq 5000)=(2.71 \pm 0.04) 10^{-3} E_{\mathrm{MeV}^{-2.12} \pm 0.07} \\
\text { photon } \mathrm{cm}^{-2} \mathrm{~s}^{-1} \mathrm{MeV}^{-1}
\end{array}
$$

As an internal check, it is noted that there is complete agreement between the high energy spectrum calculated by the saturation method and the above spectrum. The significant difference between the high and low energy spectral indices clearly shows that the time averaged Vela pulsed spectrum from 50 to $5000 \mathrm{MeV}$ cannot be described by a single power law. At least two are required and in this representation the good connection between the two spectra at $300 \mathrm{MeV}$ strongly supports the choice of $300 \mathrm{MeV}$ as the energy of the break.

The luminosity of the pulsed emission from Vela has been determined by integrating the two-power-law phase averaged spectrum for each observation. The result, displayed in Fig. 4a, corroborates the striking long-term variability of Vela's brightness presented in Sect. 3. In addition, the likelihood analysis provides simultaneously the background intensity for each observation, and its stable behaviour from one observation to the next, displayed in Fig. $4 \mathrm{~b}$, confirms the true source origin of the variability phenomenon. Further confirmation of this effect comes from the phase dependency of the variability (this shall be discussed later in this section).

To study the energy dependence of this variability, Fig. 5a shows the evolution of the pulsed flux for the energy ranges 50-300 MeV and 300-5000 MeV. The main contribution to the flux variability is clearly due to the low energy emission. The different evolutions of the flux from the two energy ranges denote a distortion of the Vela spectrum from one epoch to the next. The 
Table 4. Spectral index and flux values of the Vela pulsar emission for energy selections 50-5000 MeV (ET), 50-300 MeV (LE) and 300-5000 (HE), and for different pulsar phase intervals (Table 2) and observation periods. Fluxes are given in units of $10^{-6} \mathrm{ph} \mathrm{cm}^{-2} \mathrm{~s}^{-1}$ (phase unit) ${ }^{-1}$ and $10^{-6} \mathrm{ph} \mathrm{cm}^{-2} \mathrm{~s}^{-1}$ for the whole pulsed emission. Also given are probabilities $(\%)$ or $\chi^{2}$ values testing for no variation with phase or time

\begin{tabular}{|c|c|c|c|c|c|c|c|}
\hline $\begin{array}{l}\text { OBSERVATICN } \\
\text { PBRIOD }\end{array}$ & PBAR 1 & INTBRPULSB1 & INTBRPULSB? & PBAR 2 & TRAILER & $\begin{array}{l}\text { HHOLB PULSED } \\
\text { BMISSION }\end{array}$ & $\begin{array}{l}\text { Sp. INDBX } \\
\text { Proba. of } \\
\text { no phase } \\
\text { variation }\end{array}$ \\
\hline $\begin{array}{cc}\text { Sp.INDBX } & \text { BT } \\
2 & \mathrm{LB} \\
& \mathrm{HB}\end{array}$ & $\begin{array}{l}2.05 \pm 0.10 \\
2.15 \pm 0.25 \\
2.43 \pm 0.27\end{array}$ & $\begin{array}{l}1.67 \pm 0.11 \\
0.8 \pm 0.7 \\
2.00 \pm 0.25\end{array}$ & $\begin{array}{l}1.80 \pm 0.20 \\
2.4 \pm 0.7 \\
2.40 \pm 0.45\end{array}$ & $\begin{array}{l}1.73 \pm 0.09 \\
1.67 \pm 0.26 \\
1.87 \pm 0.18\end{array}$ & $\begin{array}{l}2.80 \pm 0.50 \\
1.9 \pm 1.5 \\
2.70 \pm 0.85\end{array}$ & $\begin{array}{l}1.84 \pm 0.06 \\
1.82 \pm 0.16 \\
2.10 \pm 0.12\end{array}$ & $\begin{array}{l}0.76 \% \\
17 \% \\
37 \%\end{array}$ \\
\hline 3 & $\begin{array}{l}2.13 \pm 0.11 \\
1.94 \pm 0.26 \\
2.87 \pm 0.39\end{array}$ & $\begin{array}{l}2.00 \pm 0.11 \\
2.00 \pm 0.25 \\
2.00 \pm 0.30\end{array}$ & $\begin{array}{l}1.91 \pm 0.16 \\
2.60 \pm 0.50 \\
1.93 \pm 0.37\end{array}$ & $\begin{array}{l}1.81 \pm 0.09 \\
1.88 \pm 0.25 \\
2.05 \pm 0.21\end{array}$ & $\begin{array}{l}2.38 \pm 0.20 \\
2.35 \pm 0.40 \\
3.1 \pm 0.9\end{array}$ & $\begin{array}{l}1.99 \pm 0.06 \\
2.03 \pm 0.14 \\
2.21 \pm 0.14\end{array}$ & $\begin{array}{l}6.6 \% \\
68 \% \\
17 \%\end{array}$ \\
\hline 12 & $\begin{array}{l}1.95 \pm 0.10 \\
1.68 \pm 0.26 \\
2.05 \pm 0.28\end{array}$ & $\begin{array}{l}1.84 \pm 0.11 \\
1.55 \pm 0.35 \\
2.17 \pm 0.25\end{array}$ & $\begin{array}{l}1.60 \pm 0.30 \\
2.5 \pm 1.0 \\
2.05 \pm 0.75\end{array}$ & $\begin{array}{l}1.78 \pm 0.10 \\
1.79 \pm 0.26 \\
2.03 \pm 0.23\end{array}$ & $2.2 \pm 1.0$ & $\begin{array}{l}1.84 \pm 0.06 \\
1.71 \pm 0.16 \\
2.07 \pm 0.14\end{array}$ & $\begin{array}{l}64 \% \\
85 \% \\
97.5 \%\end{array}$ \\
\hline 45 & $\begin{array}{l}1.80 \pm 0.11 \\
1.69 \pm 0.31 \\
1.98 \pm 0.27\end{array}$ & $\begin{array}{l}1.65 \pm 0.12 \\
0.45 \pm 0.50 \\
2.08 \pm 0.28\end{array}$ & $\begin{array}{l}1.25 \pm 0.30 \\
1.60 \pm 0.46\end{array}$ & $\begin{array}{l}1.59 \pm 0.09 \\
1.10 \pm 0.40 \\
2.00 \pm 0.20\end{array}$ & $\begin{array}{l}1.85 \pm 0.40 \\
2.8 \pm 0.8\end{array}$ & $\begin{array}{l}1.66 \pm 0.06 \\
1.23 \pm 0.22 \\
2.01 \pm 0.13\end{array}$ & $\begin{array}{l}40 \% \\
10 \% \\
78 \%\end{array}$ \\
\hline 59 & $\begin{array}{l}1.95 \pm 0.12 \\
1.35 \pm 0.30 \\
2.40 \pm 0.40\end{array}$ & $\begin{array}{l}1.71 \pm 0.11 \\
1.23 \pm 0.30 \\
1.93 \pm 0.31\end{array}$ & $\begin{array}{l}2.15 \pm 0.25 \\
2.75 \pm 0.52 \\
3.5 \pm 1.0\end{array}$ & $\begin{array}{l}1.82 \pm 0.10 \\
1.60 \pm 0.30 \\
2.39 \pm 0.28\end{array}$ & $\begin{array}{l}1.85 \pm 0.25 \\
1.38 \pm 0.58\end{array}$ & $\begin{array}{l}1.84 \pm 0.06 \\
1.52 \pm 0.15 \\
2.30 \pm 0.18\end{array}$ & $\begin{array}{l}43 \% \\
13 \% \\
38 \%\end{array}$ \\
\hline $\begin{array}{l}\text { INDRZ: } \\
\text { Tine } \\
\text { Average }\end{array}$ & $\begin{array}{l}1.98 \pm 0.05 \\
1.78 \pm 0.13 \\
2.30 \pm 0.14\end{array}$ & $\begin{array}{l}1.79 \pm 0.06 \\
1.43 \pm 0.16 \\
2.04 \pm 0.12\end{array}$ & $\begin{array}{l}1.83 \pm 0.10 \\
2.55 \pm 0.19 \\
2.11 \pm 0.22\end{array}$ & $\begin{array}{l}1.74 \pm 0.04 \\
1.66 \pm 0.12 \\
2.03 \pm 0.10\end{array}$ & $\begin{array}{l}2.25 \pm 0.15 \\
2.02 \pm 0.32 \\
2.85 \pm 0.48\end{array}$ & $\begin{array}{l}1.84 \pm 0.03 \\
1.72 \pm 0.07 \\
2.12 \pm 0.07\end{array}$ & $\begin{array}{l}0.01 \% \\
0.95 \% \\
19:\end{array}$ \\
\hline $\begin{array}{l}\text { INDBX: proba } \\
\text { no tiue } \\
\text { variability }\end{array}$ & $\begin{array}{l}31: \\
30: \\
30:\end{array}$ & $\begin{array}{l}14: \\
4 \% \\
97 \%\end{array}$ & $\begin{array}{l}21: \\
99.9 \% \\
40 \%\end{array}$ & $\begin{array}{l}39 \% \\
45 \% \\
62 \%\end{array}$ & $\begin{array}{l}17 \% \\
40 \% \\
92 \%\end{array}$ & $\begin{array}{l}0.18 \% \\
1.3 \% \\
67 \%\end{array}$ & $\begin{array}{l}0.10 \% \\
3.6 \% \\
68 \%\end{array}$ \\
\hline OBSBRVATION & PBAR 1 & INTERPULSBI & INTERPULSE? & PBAR 2 & TRAILBR & \multicolumn{2}{|c|}{ HHOLB PULSBD BMISSION } \\
\hline $\begin{aligned} \text { PLUX: } & \text { BT } \\
2 & \text { LB } \\
& \text { HB }\end{aligned}$ & $\begin{aligned} 3.8 & \pm 4.7 \\
69.5 & \pm 6.9 \\
12.8 & \pm 0.3\end{aligned}$ & $\begin{array}{l}18.6 \pm 1.2 \\
6.3 \pm 2.5 \\
6.7 \pm 0.1\end{array}$ & $\begin{array}{l}15.6 \pm 1.6 \\
13.1 \pm 4.0 \\
1.0 \pm 0.2\end{array}$ & $\begin{array}{l}55.6 \pm 2.5 \\
40.2 \pm 4.0 \\
12.7 \pm 0.15\end{array}$ & $\begin{array}{l}11.6 \pm 2.2 \\
2.8 \pm 2.0 \\
2.6 \pm 0.25\end{array}$ & $\begin{array}{l}20.5 \pm 1.5 \\
13.5 \pm 2.3 \\
1.68 \pm 0.15\end{array}$ & \\
\hline 3 & $\begin{array}{l}92.4 \pm 6.0 \\
73.6 \pm 8.0 \\
10.8 \pm 1.0\end{array}$ & $\begin{array}{l}45.7 \pm 3.0 \\
42.5 \pm 4.7 \\
4.7 \pm 0.1\end{array}$ & $\begin{array}{l}28.1 \pm 2.5 \\
31.7 \pm 6.0 \\
4.1 \pm 0.1\end{array}$ & $\begin{array}{l}71.8 \pm 4.2 \\
56.6 \pm 6.5 \\
14.6 \pm 0.6\end{array}$ & $\begin{array}{l}25.3 \pm 2.5 \\
23.6 \pm 3.6 \\
1.5 \pm 0.2\end{array}$ & $\begin{array}{l}32.3 \pm 2.3 \\
28.7 \pm 3.7 \\
4.2 \pm 0.2\end{array}$ & \\
\hline 12 & $\begin{array}{l}72.8 \pm 4.0 \\
57.1 \pm 6.7 \\
9.3 \pm 0.2\end{array}$ & $\begin{array}{l}26.6 \pm 1.8 \\
15.5 \pm 2.6 \\
6.6 \pm 0.2\end{array}$ & $\begin{array}{l}4.6 \pm 0.7 \\
6.5 \pm 2.6 \\
0.66 \pm 0.05\end{array}$ & $\begin{array}{l}49.2 \pm 2.5 \\
37.6 \pm 4.6 \\
10.4 \pm 0.25\end{array}$ & $0.7 \pm 0.4$ & $\begin{array}{l}16.8 \pm 1.1 \\
12.4 \pm 1.9 \\
3.18 \pm 0.05\end{array}$ & \\
\hline 45 & $\begin{array}{l}61.9 \pm 4.0 \\
50.5 \pm 7.5 \\
10.1 \pm 0.2\end{array}$ & $\begin{array}{l}19.0 \pm 1.3 \\
6.2 \pm 3.1 \\
5.2 \pm 0.1\end{array}$ & $\begin{array}{l}2.9 \pm 0.5 \\
2.92 \pm 0.05\end{array}$ & $\begin{array}{l}52.6 \pm 2.5 \\
24.7 \pm 5.4 \\
17.0 \pm 0.25\end{array}$ & $\begin{array}{l}3.0 \pm 0.7 \\
1.4 \pm 0.15\end{array}$ & $\begin{array}{l}15.1 \pm 0.9 \\
7.9 \pm 1.8 \\
4.3 \pm 0.1\end{array}$ & \\
\hline 59 & $\begin{array}{l}87.6 \pm 5.0 \\
59.6 \pm 9.2 \\
10.2 \pm 0.4\end{array}$ & $\begin{array}{l}42.3 \pm 2.2 \\
31.4 \pm 5.0 \\
5.0 \pm 0.1\end{array}$ & $\begin{array}{l}27.1 \pm 3.0 \\
34.2 \pm 5.6 \\
1.0 \pm 0.2\end{array}$ & $\begin{array}{l}72.1 \pm 3.5 \\
49.7 \pm 6.7 \\
15.0 \pm 0.3\end{array}$ & $\begin{array}{l}9.2 \pm 1.1 \\
8.7 \pm 2.6\end{array}$ & $\begin{array}{l}28.1 \pm 1.8 \\
22.3 \pm 3.7 \\
3.5 \pm 0.1\end{array}$ & \\
\hline $\begin{array}{l}\text { PLUX: } \\
\text { Tine } \\
\text { Average }\end{array}$ & $\begin{array}{l}78.7 \pm 2.1 \\
60.6 \pm 3.5 \\
10.4 \pm 0.2\end{array}$ & $\begin{array}{l}30.6 \pm 0.9 \\
20.2 \pm 1.7 \\
5.62 \pm 0.06\end{array}$ & $\begin{array}{l}15.0 \pm 0.9 \\
16.8 \pm 2.2 \\
2.25 \pm 0.07\end{array}$ & $\begin{array}{l}60.0 \pm 1.4 \\
41.1 \pm 2.6 \\
14.0 \pm 0.2\end{array}$ & $\begin{array}{l}8.7 \pm 0.6 \\
6.2 \pm 1.0 \\
0.9 \pm 0.2\end{array}$ & $\begin{array}{l}22.1 \pm 0.7 \\
16.5 \pm 1.3 \\
3.89 \pm 0.06\end{array}$ & \\
\hline $\begin{array}{l}\text { PLUX: } \\
\text { Chi² no tine } \\
\text { variability }\end{array}$ & $\begin{array}{l}27 \\
7 \\
108\end{array}$ & $\begin{array}{l}191 \\
73 \\
298\end{array}$ & $\begin{array}{l}261 \\
38 \\
900\end{array}$ & $\begin{array}{l}50 \\
16 \\
274\end{array}$ & $\begin{array}{l}187 \\
92 \\
114\end{array}$ & $\begin{array}{l}103 \\
38 \\
94\end{array}$ & \\
\hline
\end{tabular}




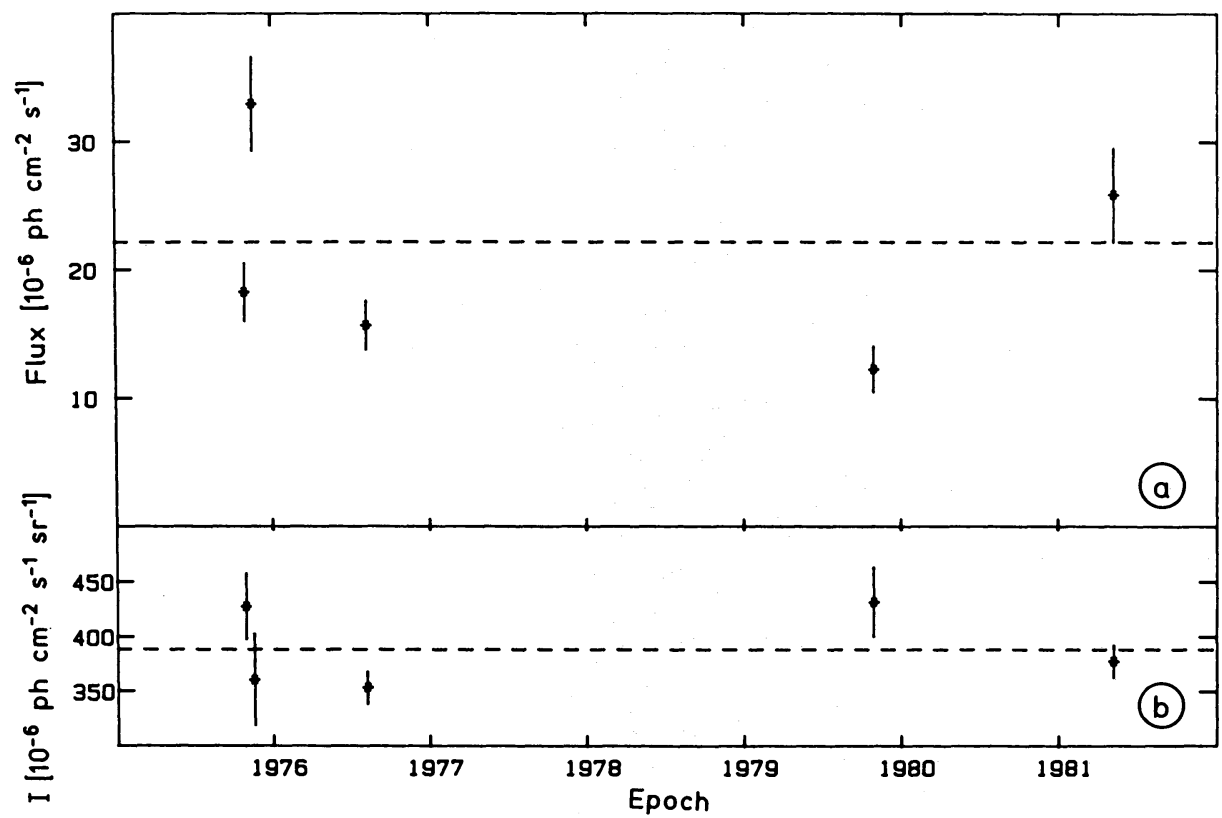

Fig. 4a and b. Integrated gamma ray flux/intensity $(I)$ values $(50-5000 \mathrm{MeV})$ calculated using the likelihood analysis for 5 observations from 1975 to 1982 . a The pulsed gamma ray flux for PSR 0833-45; b the diffuse galactic background and instrumental emission

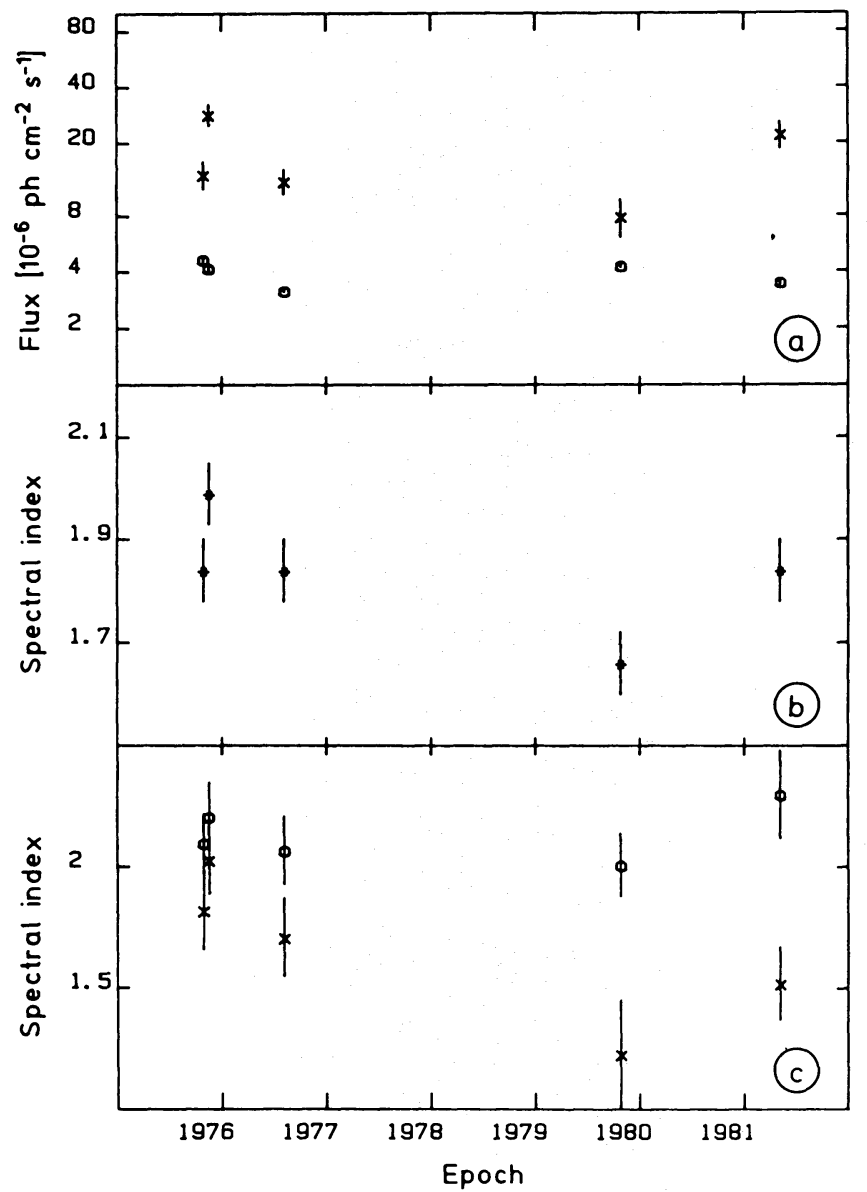

Fig. 5. a Vela pulsed gamma ray flux values integrated over 50-300 MeV $(\times)$ and $300-5000 \mathrm{MeV}\left({ }^{\circ}\right)$ for each observation period; b phase averaged pulsed spectral index over the total energy range $(50-5000 \mathrm{MeV})$; c phase averaged pulsed spectral indices for high $\left({ }^{\circ}\right)$ and low $(x)$ energy components observed fluctuation in the spectral ratios (the ratio of low to high energy flux) has a probability of $10^{-10}$ of being due to a random effect. Figures $5 \mathrm{~b}$ and $5 \mathrm{c}$ show the spectral indices as a function of epoch for the 3 energy ranges. As expected, the spectral index for the entire energy range is seen to vary with time with a probability of $210^{-3}$ that this effect is due to chance ${ }^{1}$, whereas a simultaneous analysis of the background spectra shows no indication for variability. The spectral variability corresponds to an overall steepening of the spectrum when the Vela flux is high, and to a flattening when the flux is low. The correlation still holds when the low energy part of the spectrum is considered, see Fig. 5c, although the effect is less significant due to the reduced number of photons in this smaller energy range (chance probability of $1.310^{-2}$ ). For the high energy part the time averaged spectral index of $2.12 \pm 0.07$ is statistically consistent with each observation but a similar trend is seen. Finally, the systematic difference between the spectral slopes for the two adjacent energy intervals is clearly observed in Figure 5c.

To study the phase dependence of the pulsed emission, the likelihood analysis has been performed independently for the 5 phase intervals defined in Table 2. From the study of flux ratios from observations 2, 3 and 12, Kanbach et al. (1980) found evidence that the second peak and the total interpulse exhibit a harder spectrum than the first peak, but they were unable to quantitatively measure these differences. Figure $6 \mathrm{a}$ shows the time averaged spectral index as a function of phase. The probability that the emission may be best represented by a homogeneous spectrum over all phases $\left(E^{-1.84}\right)$ is $10^{-4}$. Although, ideally, a single power law is not the best fit to the individual phase regions,

\footnotetext{
${ }^{1}$ Although it has been established that the Vela spectrum cannot be described by a single power law over the COS-B energy range, the comparison of the evolution of the spectral index for the single power law fit is used to support the evidence for spectral variability during the observations. Obviously, this test of variability is less sensitive than the variation in spectral ratio.
} 


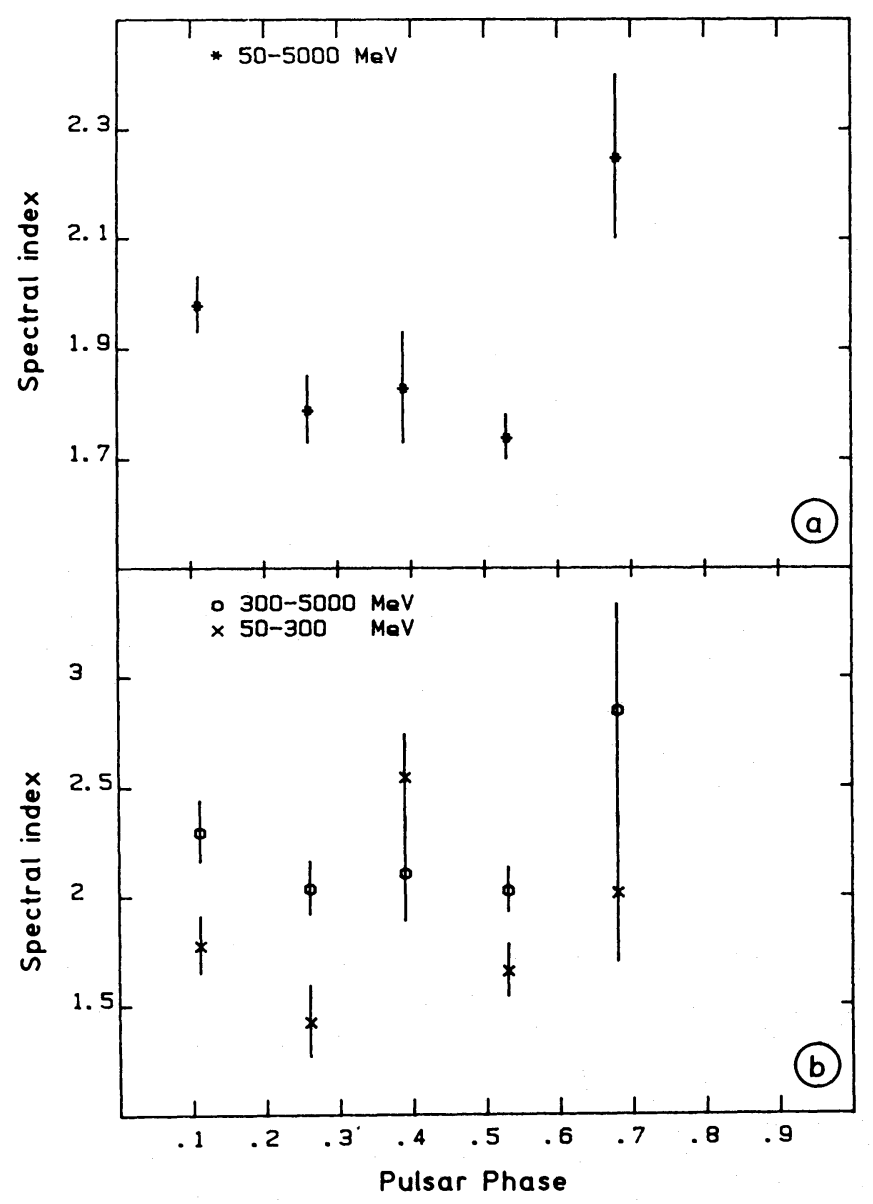

Fig. 6a and b. Time averaged pulsed spectral index as a function of phase; a one power law fit over total energy range; $\mathbf{b}$ for high and low energy ranges

the result confirms the spectral differences noted by Kanbach et al. (1980). The second peak is much harder than the first $(3.75 \sigma)$, as are to a lower degree of significance the interpeak emissions, while the trailer is seen to exhibit an extremely soft spectrum. A further understanding of the phase dependency of the pulsed emission may be obtained from Fig. 6b, which shows the spectral characteristics of the high and low energy emissions as a function of phase. Although the existence of a spectral break in the whole pulsed emission has been clearly established in the above results, it is not possible, due to the reduced statistics, to draw such a firm conclusion for each phase interval separately. In all cases, the difference between the indices from the low and high energy ranges lies within the 2.5 to $3.0 \sigma$ level. This is not statistically significant, but it strongly indicates that each of the phase components have curved spectra.

Analysis of the spectral characteristics of the high energy emission shows that there is no significant variation in its spectral index with phase, giving a phase averaged value of $2.12 \pm 0.07$. Thus, the spectral index of the $\mathrm{GeV}$ radiation from Vela displays both a stable and homogeneous behaviour. In contrast, the spectral index of the low energy emission shows evidence for phase dependence (chance probability $510^{-3}$ ). It is evident from the change in the spectral index at low energies (from the first peak to interpulse- $2,4.5 \sigma$, and interpulse- 2 to the second peak, $3.6 \sigma$ ) and from the softness of the first peak and trailer over the entire energy range, that 5 separate phase components exist in the Vela gamma ray lightcurve. Unfortunately, due to limited statistics, the boundaries of the phase components could not be treated as additional free parameters. However, the selected boundaries are found to be in a good agreement with the results of a cluster analysis study of the Vela lightcurve topology using observation period 2 by Buccheri et al. (1987). The two approaches (spectral and topological) concur to conclude that the pulsar gamma ray emission is composed of discrete components.

Figure 7 shows the evolution of the flux from each of the phase components for each, observation and for both high and low energy ranges. The long-term trend is obviously similar for each phase component, however the relative amplitude of the variability is significantly larger for the two interpulse emissions. In the low energy range, the flux from interpulse- 1 is seen to increase by a factor of 7 within 2 weeks while the first peak remains stable during the same interval. This phase dependent effect gives weight to the hypothesis that the variability truely originates from Vela and is not an instrumental effect. It is of interest to investigate whether the flux variations in the separate energy intervals are the result of spectral distortion or are purely changes in absolute flux. Unfortunately, the low statistics reduce the sensitivity and no significant spectral index variability is observed for any of the phase components, see Table 4. Figure 8 shows the spectral ratios,

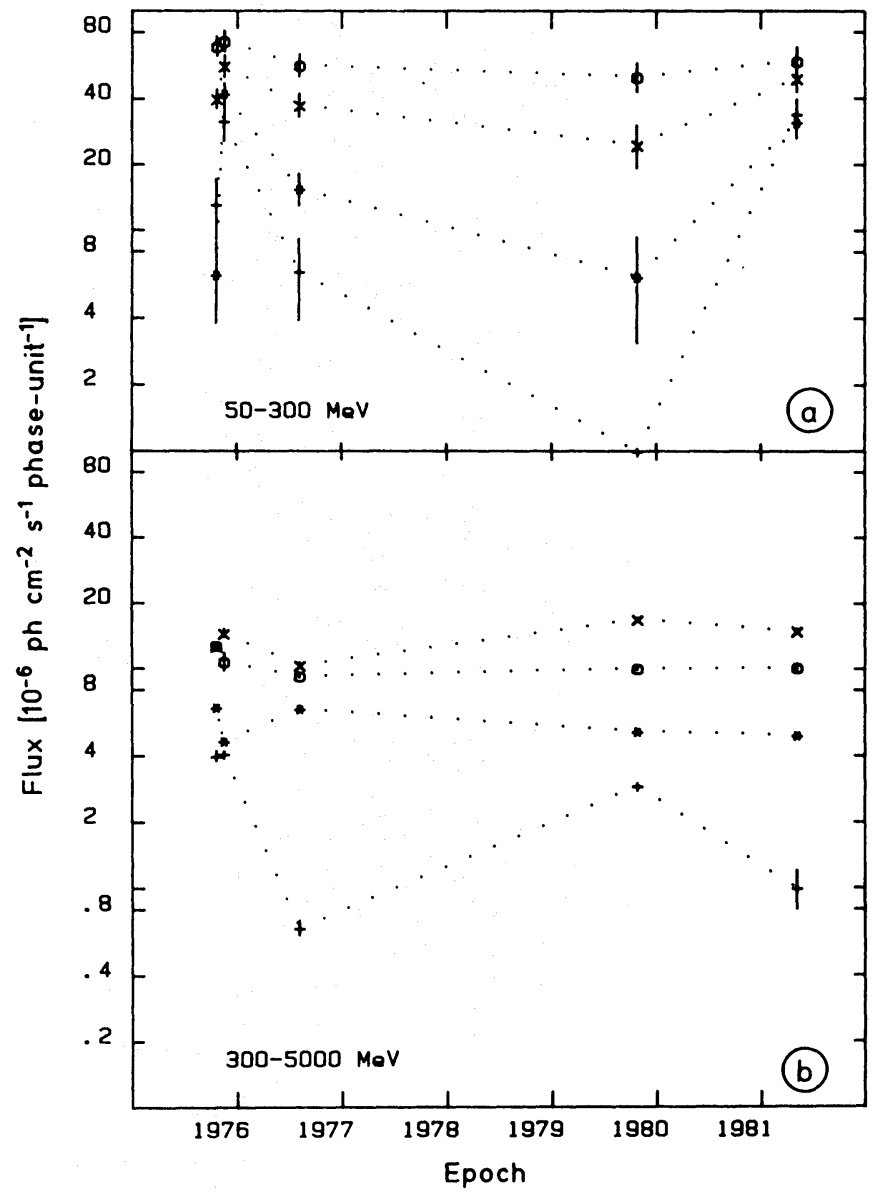

Fig. 7a and b. Integrated gamma ray flux from each phase component as a function of epoch, a for low energy range, $\mathbf{b}$ for high energy range ( $\circ$ peak $1, *$ interpulse- $1,+$ interpulse $-2, \times$ peak 2 ) 


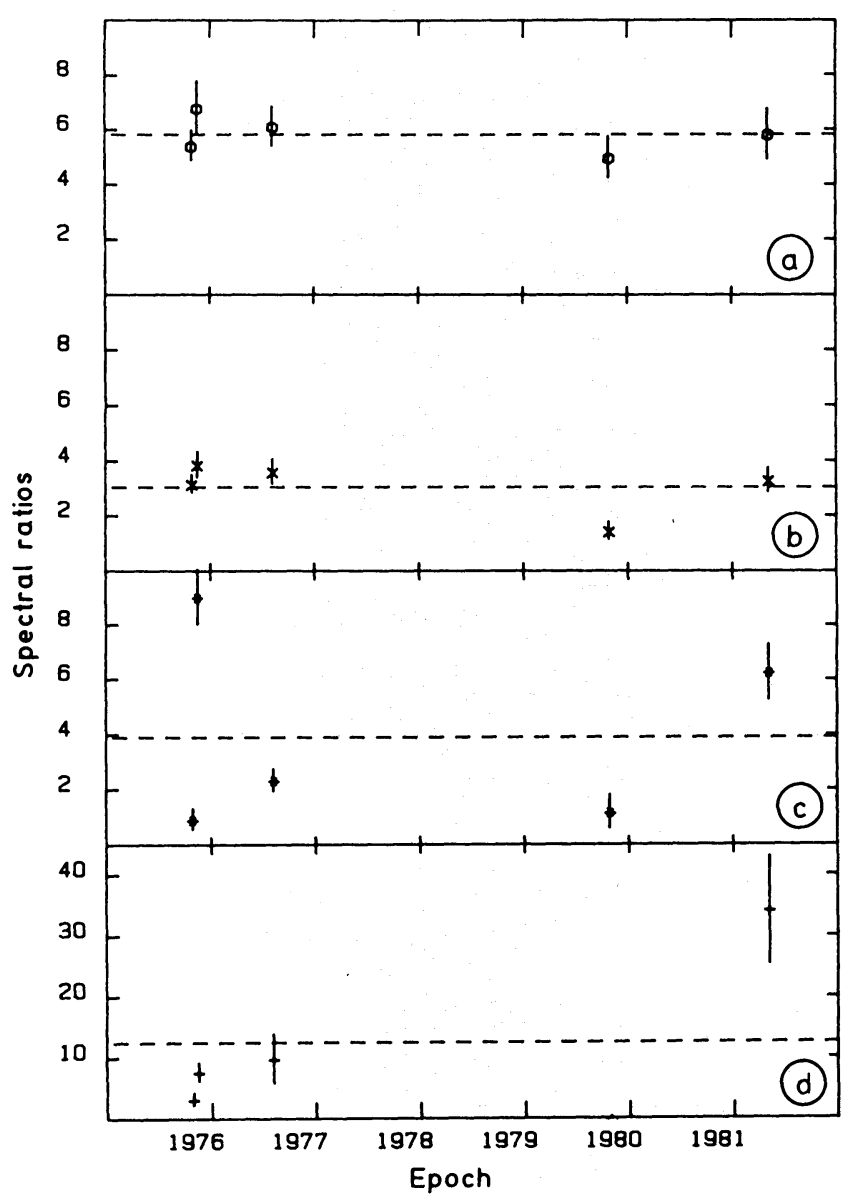

Fig. 8a-d. Ratio of low to high energy flux as a function of epoch; a peak 1 ; b peak 2 ; $\mathbf{c}$ interpulse-1; $\mathbf{d}$ interpulse-2

calculated from the fluxes derived for the high and low energy ranges, as a function of epoch for each phase component (for the trailer the statistics are too poor to produce a meaningful result). For the first peak, no significant alteration with time in the relative spectral properties from the two energy ranges is observed (chance probability 0.56 ). For the remaining phase components, a significant distortion of the spectrum is detected (chance probability interpulse-1: $<10^{-25}$, interpulse-2: $10^{-20}$ and peak 2 : $\left.710^{-6}\right)$.

To illustrate their long term evolution, the energy spectra giving the maximum likelihood fits to the data for each phase component and for each observation are displayed in Fig. 9. Good agreement is observed between the low and high energy fits at approximately $300 \mathrm{MeV}$ for most phase components. The two power law representation is therefore a good approximation to the real spectra (for the trailer, the poor statistics make interpretation of the results rather difficult, therefore the reader is referred to the single power law fit in Table 4 to judge the trailer evolution). The actual position of the break or bend in the real spectrum will vary somewhat in energy following the apparent long term variability. The phase averaged spectra, Fig. 9f, show the high energy emission to be rather stable throughout the observations, while the low energy emission is seen to vary significantly and is responsible almost entirely for the observed flux variability.
In conclusion, the results of the analysis show the Vela pulsar as a most complicated gamma ray source. The emission is found to be highly variable with time. Five distinct phase components are identified which are individually variable and show different spectral characteristics. The energy spectrum over the $50-5000 \mathrm{MeV}$ region is best represented by a two power law fit with a spectral break at approximately $300 \mathrm{MeV}$. The observed variability is found to be almost entirely due to spectral changes in the low energy part of the emission with the high energy component remaining comparatively stable throughout the period of the observations.

\section{Discussion}

\subsection{Total PSR 0833-45 spectrum}

The two-power-law-fit time averaged spectrum of the whole pulsed emission has been plotted in Fig. 10 together with the data recorded at other wavelengths in order to show the full energy distribution of the Vela pulsed radiation over the entire electromagnetic spectrum. Over this spectrum, the pulsar reaches its maximum luminosity in the $\mathrm{MeV}-\mathrm{GeV}$ domain. At very high energies, the recent results of Bhat et al. (1987) indicate a spectral slope of -3.5 at $\mathrm{TeV}$ energies which does not require an excessive steepening when extrapolating over three decades of energy from the COS-B gamma ray range. The observed time variability presented in this article may support the proposed time variability at TeV energies, e.g. Bhat et al. (1980), however, it should be noted that the variations recorded above a few hundred $\mathrm{MeV}$ are relatively small $(\leq 25 \%)$.

In order to take into account the large time variability at lower energies, the gamma ray spectra derived in the extreme cases of periods 3 and 45 have also been displayed in Fig. 10. The result between 35 and $100 \mathrm{MeV}$ obtained by SAS-2 in February and April 1973, is in good agreement with the COS-B time averaged spectrum, as is the spectrum derived by Tümer et al. (1984) in November 1981 at lower energies (1-30 MeV). Their lowest energy point $(0.3-1.5 \mathrm{MeV})$ together with the monotoneous increase in the spectral index from $1 \mathrm{MeV}$ to $5 \mathrm{GeV}$ strongly suggest that the shape of the whole pulsed energy distribution in the gamma ray domain is a smooth curve. The very low upper limits set by the Einstein and HEAO 1 satellites (energy ranges $0.1-4.5$ and $15-175 \mathrm{keV}$ ) suggest that the energy distribution in the $\mathrm{X}$-ray domain is still bending down before rising again towards the optical and radio wavelengths. Since the pulsed X-ray emission may also be time variable, the bulk of the other negative detections has been added to the figure as well. The relatively low X-ray emissivity of the Vela pulsar is in clear contrast to that of the Crab pulsar. The latter exhibits an X-ray luminosity which is about equal to that in the gamma ray domain from $1 \mathrm{MeV}$ to $1 \mathrm{GeV}$. This difference might indicate different physical or geometrical conditions in the magnetosphere.

\subsection{Long-term time variability of PSR 0833-45}

The flux evolution presented in Figs. 2 and 4 demonstrates the gamma ray variability of the pulsar over several years. The abrupt increase in luminosity observed during the first two periods indicates that the variations may also occur on a much shorter time-scale. Figure 11 presents a close-up view of this epoch where the first two observations have been sub-divided into time 

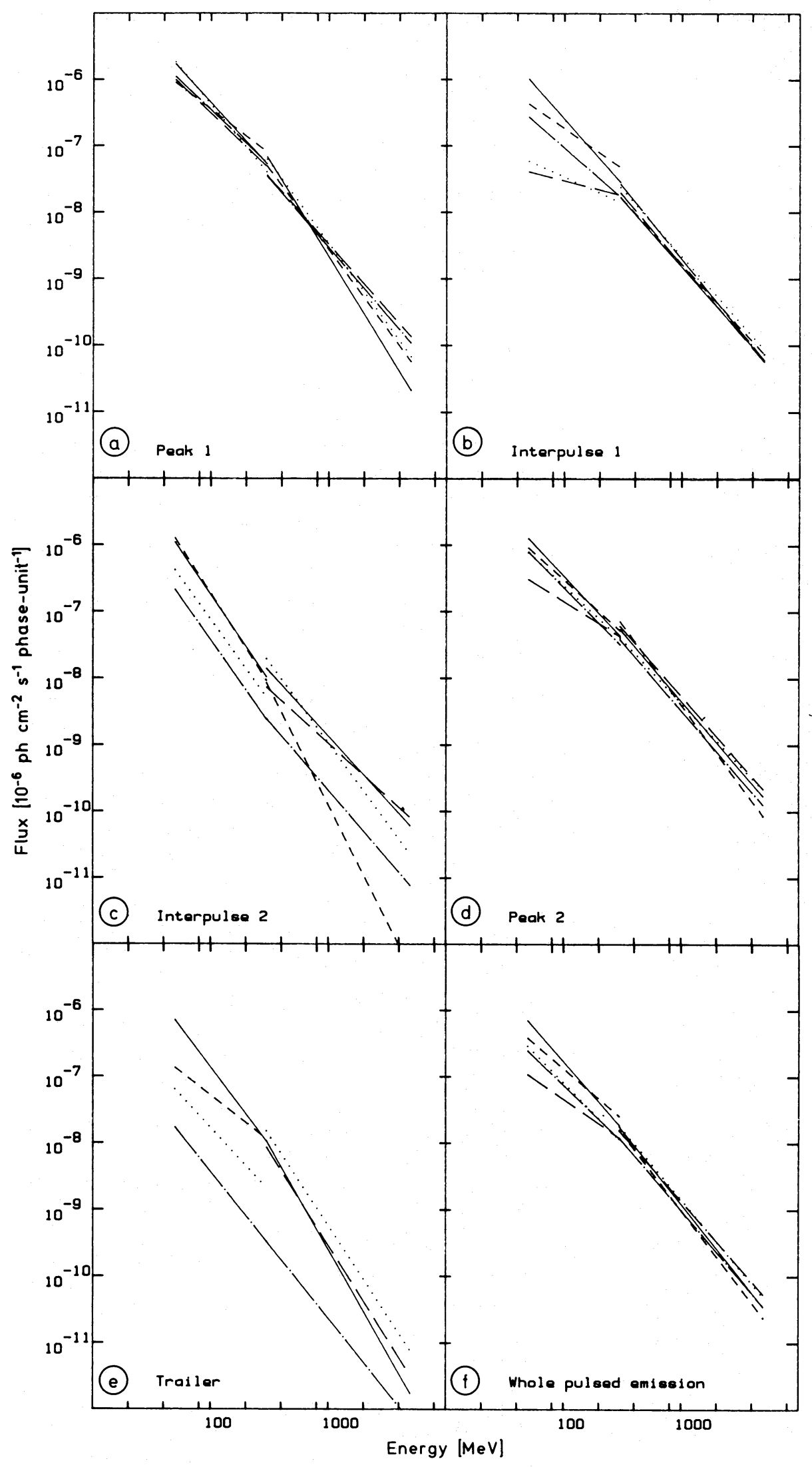

Fig. 9. Differential pulsed gamma ray spectra $(50-5000 \mathrm{MeV})$ from Vela for 5 phase intervals and the total phase averaged emission. The power law spectra giving the maximum likelihood fits are shown for the $50-300 \mathrm{MeV}$ and $300-5000 \mathrm{MeV}$ energy ranges, respectively, for each observation; period $2 \ldots \ldots$, period $3-$, period $12-\cdot-\cdot$, period $45-\ldots$, period $59---$ 


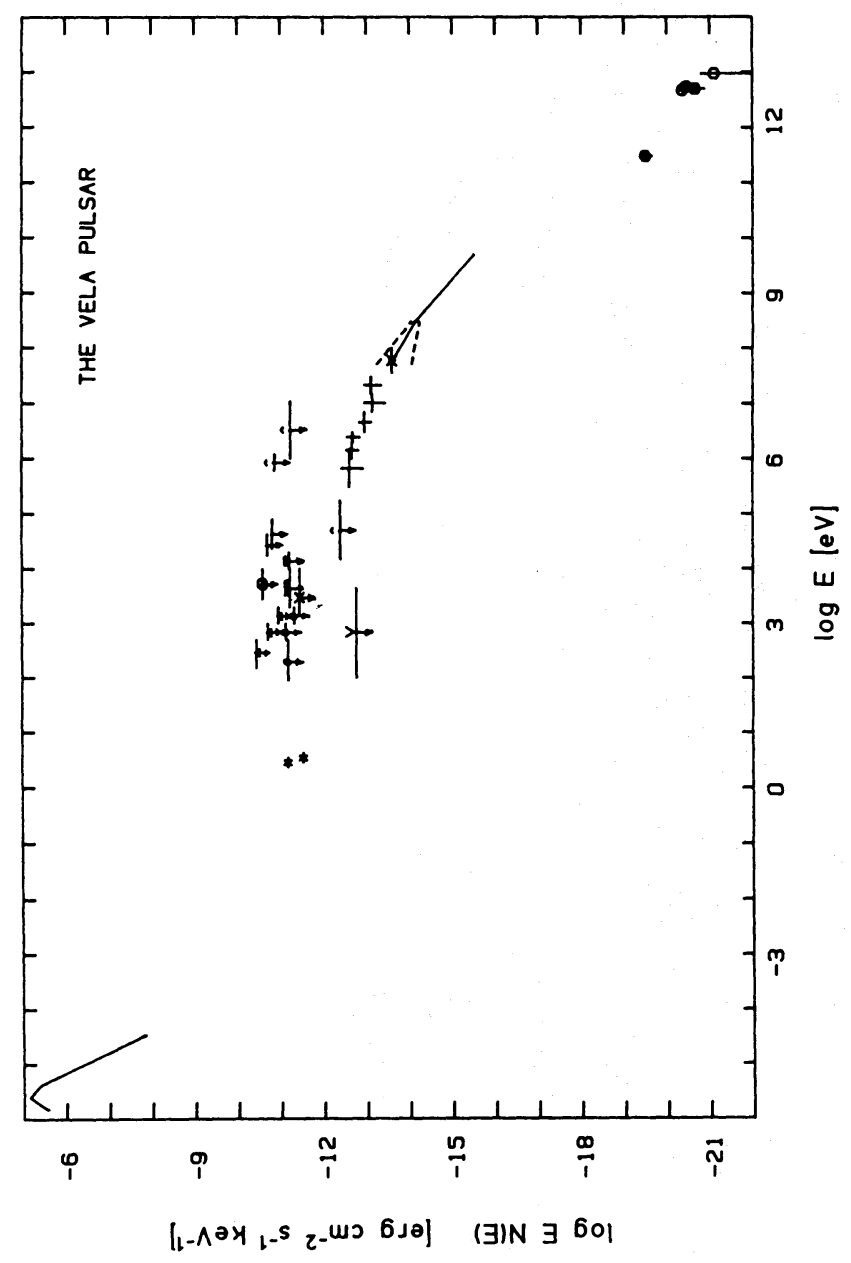

intervals of about 10 days. The corresponding fluxes have been estimated using the "saturation method" to derive the count rates, and the telescope sensitivity averaged over the full observation period. Strictly taken, the latter might not be correct, as the sensitivity might fluctuate within one observation period. However, the COS-B behaviour has proved to be very stable during the first four periods (only a $3 \%$ change in sensitivity over the first four months after launch, Strong et al., 1987a). To quantify the time scales of the evolution, two exponential functions have been fitted to these data and are displayed in Fig. 11. The resultant rise and decay times are $11.4 \pm 1.0$ and $137 \pm 39$ days respectively, which means that the pulsar high energy behaviour evolved on a time-scale of about 10 days and that the effect lasted up to a few months. According to the fit, the maximum flux was reached on November 16 1975, that is about one month after a large discontinuity in the pulsar rotational parameters, a so-called glitch. The glitch occurred sometime between September 25 and October 15 (Manchester et al., 1976), while the gamma ray observations started on October 20. Although the exponential function starts rising at the epoch of the glitch, one has to keep in mind that the exponential model is rather arbitrary and has no a priori physical support. The decay time of the gamma ray perturbation differs significantly from the rotational one, which was found to be 640 days (Kanbach et al., 1980). This difference is not surprising since the gamma ray decay corresponds to a magnetosphere-reaction time scale while the rotational decay time is linked to the relaxation of the inner star phenomena. From an energy point of view, the relationship between the two events is not excluded. Assuming that the pulsar energy stockpile is the kinetic energy of rotation, the spin down luminosity is of the order of $10^{37} \mathrm{erg} \mathrm{s}^{-1}$ (using a typical moment of inertia of $1.4-210^{45} \mathrm{~g} \mathrm{~cm}^{2}$, Shapiro and Teukolsky (1983). The increase in luminosity caused by the glitch just after its occurrence amounts to $0.8-1 \%$ of this value (Manchester et al., 1976; Downs, 1981). On the other hand the power radiated through the $50-5000 \mathrm{MeV}$ gamma rays during the "quiet" behaviour of the pulsar is of the order of $10^{35} \mathrm{erg} \mathrm{s}^{-1}$ (assuming that Vela is $500 \mathrm{pc}$ away and that it radiates over one pole), and the gamma ray outburst observed after the glitch corresponds to an additional output of approximately $0.410^{35} \mathrm{erg} \mathrm{s}^{-1}$ radiated by the pulsar at its maximum luminosity. These approximate values indicate that if the gamma ray flare is related to the glitch event, then the burst observed by COS-B in 1975 required initially an appreciable part of the increase in rotational energy release caused by the pulsar spin-up. However, the recurrence of such a phenomenon may be questioned. A further giant glitch occurred in July 1978 (Downs et al., 1978) when no COS-B data were taken from the source (see Fig. 2). According to the time scales quoted above, a coincident gamma ray flux increase could have happened without being noticed in later COS-B observations. What is surprising is the high gamma ray flux recorded in April and May 1981 when no glitch was reported. Such an event occurred five months later, in October 1981 (Gorenstein et al., 1981), when Vela was not in the field-of-view. Thus, the gamma ray enhancement is recurrent but it does not necessarily follow a glitch event. For this reason, it is not possible to draw any firm conclusion from the COS-B data on the relationship between the gamma ray variability and the glitch phenomenon.

The Vela pulsed radio flux is not stable either. As shown in Fig. 12, three large irregularities were recorded at $408 \mathrm{MHz}$ between 1971 and August 1978 when the observations at the 


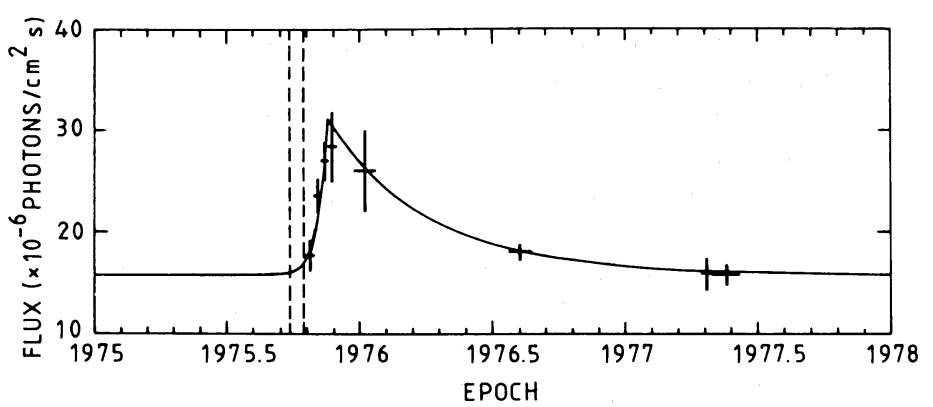

Fig. 11. Expanded view of the high energy gamma ray flux from the Vela pulsar. Observations periods 2 and 3 have been sub-divided into 10 day intervals to study the short term structure of the variability. The solid line represents two 'back to back' exponential functions fitted to the data. The dashed vertical lines represent the uncertainty in the epoch of a large discontinuity in the pulsar period (Manchester et al., 1976)

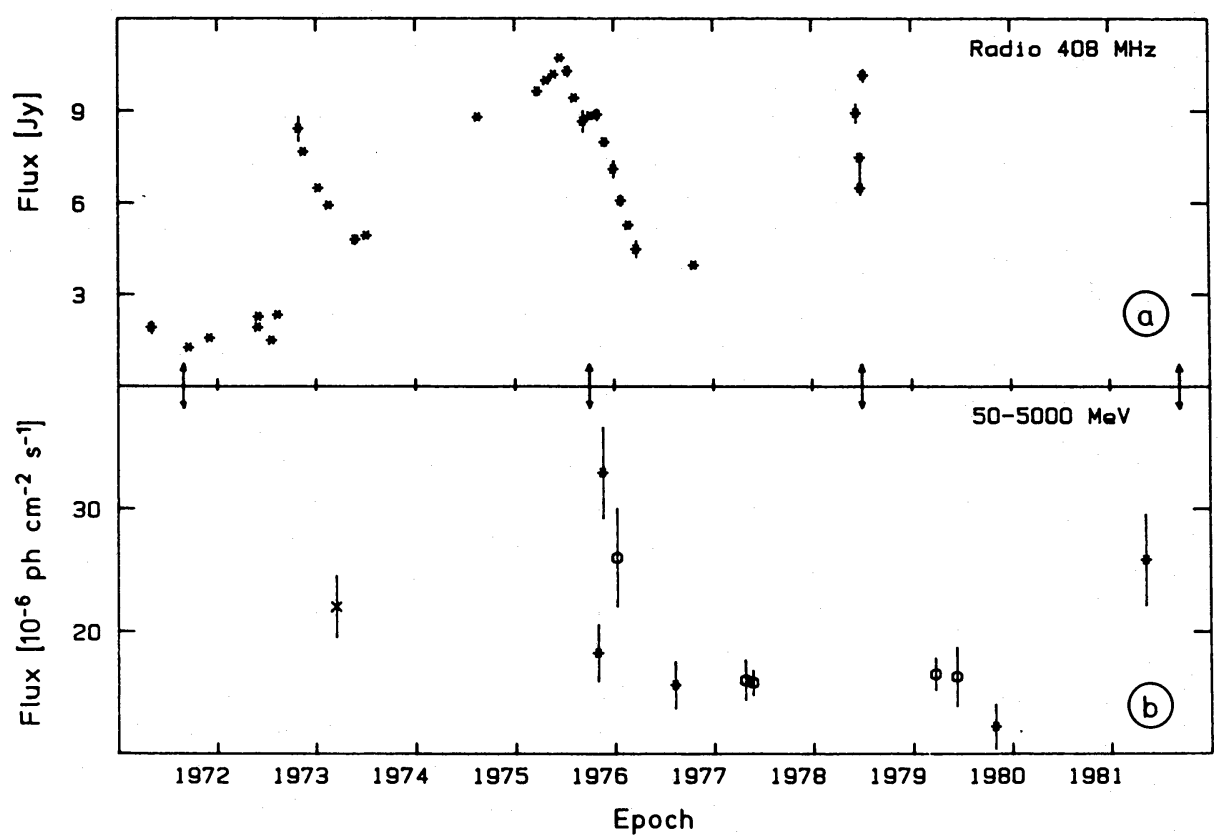

Fig. 12a and b. Comparison between the evolution of a the radio flux at $408 \mathrm{MHz}$ (McAdam, 1981) and b the high energy gamma ray flux for the Vela pulsar. Gamma ray data points: * COS-B likelihood analysis; - COS-B saturation method; +SAS-2 (Thompson et al., 1977). The epochs of the pulsar glitches are indicated by arrows
Molonglo Observatory were stopped (McAdam, 1981). Unfortunately, additional $408 \mathrm{MHz}$ data are not available beyond this date, but further studies at $843 \mathrm{MHz}$ seem to corroborate the long term radio variability (McAdam, private communication). These secular changes could not be explained by the interstellar scintillation, nor do they appear to be related to the glitches. Their rather regular aspect over the years suggested that such fluctuations are caused by a precession in the beam angle with respect to our line of sight (McAdam, 1981). Despite the minimal overlap of the radio and the gamma ray data, one could conclude that the two variation patterns are not strongly correlated. The small irregularity in the general radio decrease, in late 1975, seems more related to the third Vela glitch (the two eccentric radio points were recorded at the beginning and the end of October) than to the gamma ray flux, which peaked more towards mid-November. Therefore, the precession effect proposed for the radio evolution is not supported by the gamma ray data, since it would not explain the stability of the first peak while the other phase components all vary. It would also be difficult in this case to account for the energy dependence of the variability (merely the 'steadiness' of the high energy part of all the different components). No other clues to the origin of the long-term variability can be found from the pulsed emission at other wavelengths. In the optical band, the 24th magnitude Vela object is too faint to provide so far precise information on possible apparent magnitude changes. Such information is very important and should become available soon. In the X-ray domain, the only long-term data are obtained with the Einstein satellite. Ten observations were made in the period from November 1978 to June 1980, during a quiet phase in the gamma ray activity but including the 1978 radio glitch. No pulsations were observed and the X-ray point source coincident with the pulsar position showed no significant variation in its flux.

Other radio pulsars have proved to be variable sources, but usually on a much shorter time-scale. In the Crab case, after a continuous weakening of the $74 \mathrm{MHz}$ pulsed emission was observed between 1971 and 1975, its radio flux remained steady over the next six years (Rickett and Seiradakis, 1982), as did its optical and X-ray flux (Knight, 1982). Throughout the COS-B observations, spread over seven years, only a marginal change in the ratio of the two pulses has been observed (Clear et al., 1987). Hence, in the long term, the Vela and Crab pulsars do behave differently and the large variability observed from Vela, both in radio and gamma rays, looks unusual compared to the measured behaviour of all other pulsars.

\subsection{Discrete components of the lightcurve}

The present results lead to the identification of at least five discrete components of the Vela pulsed gamma ray emission. These exhibit different spectral distributions at low energies and 
different long term evolutions. Of particular interest is the interpulse radiation which is found to be composed of two discrete phase components, with the first being approximately coincident in phase with the first optical peak. Such a phase coincidence is not clear in the case of interpulse- 2 and gamma ray emission in phase with the second optical peak would largely disappear beneath the strong second gamma ray peak (see Fig. 1). Furthermore, while the two optical pulses exhibit a similar structure in phase and have similar luminosities and colours (Peterson et al., 1978), interpulse-1 shows a broad peak and is brighter than the flat interpulse- 2 and they clearly have different spectral characteristics. The spectrum of interpulse- 1 exhibits a pronounced break around $300 \mathrm{MeV}$ and highly variable low energy emission. In contrast, the interpulse- 2 emission seems to present a surprisingly "convex" energy distribution. Unfortunately, it is impossible to conclude on the reality of such a reversed shape because the discrepancy between the average low and high energy spectral indices does not reach a $2 \sigma$ significance level. It is striking, however, that the remarkably steep distribution at low energies is present during four independent observations (the interpulse- 2 radiation during period 45 was too faint to be analysed). The high values of the interpulse- 2 spectral ratios corroborates the extreme softness of its radiation. The SAS-2 data, at energies above $35 \mathrm{MeV}$, may also support this characteristic. In the lightcurve of Thompson et al. (1977) the flux from the two interpulse phase regions is about equal. This relative increase of the interpulse- 2 component may be explained by the extreme softness of its spectrum as measured in the COS-B data. Unfortunately, it is not possible from the two-power-law representation that has been adopted here to know whether the steep low energy part starts flattening around $50 \mathrm{MeV}$. The relative fluxes of the interpulse- 2 and the second peak as observed by SAS- 2 and extrapolated from COS-B do not suggest such a bending. However, a sharp turn-over must exist around a few tens of $\mathrm{MeV}$, otherwise interpulse- 2 would be clearly visible in the soft gamma ray lightcurve of Tümer et al. (1984), see Fig. 1. As expected, the extrapolation of the harder interpulse- 1 spectrum to medium gamma ray energies (1-30 MeV) disappears within the statistical fluctuations of the lightcurve. Further observations in this energy range leading to a precise lightcurve are of importance for the study of the interpulse emissions and their physical origins. Such observations will be provided by the COMPTEL experiment onboard the Gamma Ray Observatory which is due for launch in 1990.

At $\mathrm{TeV}$ energies the detection of pulsed gamma rays at phase 0.27 (Bhat et al., 1987) indicates that the interpulse-1 phase component, which is by far the most variable part, could also be related to the very high energy gamma ray activity of the pulsar. Further observations at these energies are also required to determine the complete gamma ray spectrum of the interpulse emission.

\subsection{Implications for Vela models}

After the demonstration by Goldreich and Julian (1969) that a pulsar magnetosphere may be filled with plasma, various models have been proposed to explain how pulsed radiation can be produced at different wavelengths within such magnetospheres. Many agree on the existence of accelerating regions called "gaps", which arise from a local charge depletion and provide the primary population of ultra relativistic particles streaming along the magnetic field lines. However, the models disagree on the location of the active regions (e.g. polar gaps, outer gaps etc.), on the type of physical processes and on the sequence of processes which convert the primary particles into pulsed radiation. An important consequence of the new description of the Vela gamma ray behaviour is that the activity in its magnetosphere is much more complex than imagined by these models. The identification in the lightcurve of discrete high energy components with their own evolution implies that several active sites exist simultaneously in the magnetosphere, and the different spectral characteristics of the components indicate that the physical processes differ with location. It is beyond the scope of this article to test the numerous pulsar models against our results. However, as several discuss the high energy emission in more detail and provide useful tests to evaluate interesting ideas, some of these shall be briefly considered.

Salvati (1986) has recently pointed out that secondary synchrotron emission would produce a spectral shape in the soft gamma ray domain which bears some information on the source location in the magnetosphere if the secondary particles originate in magnetic photoabsorption. The predicted synchrotron spectrum has a slope of -1.5 between two characteristic photon energies. This slope is consistent with the data reported by Tümer et al. (1984) but the upper break is not visible in Fig. 10. The new COS-B spectrum suggests that the total gamma ray spectrum is a smooth curve, continuously steepening with increasing energy. Adopting the upper break energy at either $15 \mathrm{MeV}$ (following Salvati) or $300 \mathrm{MeV}$ (as suggested by the COS-B data), the test leads in both cases to a source location extremely close to the star surface and in complete contradiction with the models that are discussed below. Moreover, one should be very cautious comparing the soft and hard gamma ray data, since the spectral variability observed below $300 \mathrm{MeV}$ may extend down to the soft gamma ray range. Although the main peaks are more stable than the interpulses, contemporary data at different energies would be preferable for future discussions on the details of the spectral shape.

A spectral break would not be predicted in the soft gamma ray range if the assumption of magnetic photoabsorption is not relevant in the Vela case, as mentioned by Cheng et al. (1986b). In the frame-work of their outer gap model, the primary photons and the secondary pairs are produced respectively by inverse Compton scattering and collisions with a tertiary population of soft synchrotron photons (mainly IR) which illuminate the outer magnetosphere. The predicted pulsed spectrum is in good agrement with Fig. 10. In the gamma ray domain, the spectral shape is in good agreement with the data of Tümer et al. (1984) and with the time averaged COS-B spectrum up to $3 \mathrm{GeV}$ where their model induces a break which is not seen in the COS-B data (see Fig. 3). Although Cheng et al. do not present any lightcurve, they calculate that their model can reproduce the different gamma ray and optical phase separations between the peaks, since the tertiary optical photons are emitted somewhat closer to the star (about half way) than the secondary gamma rays. However, the outer gap model cannot explain the existence of the two gamma ray interpulse components. Because of their phase position, they should also originate from a part of the gap closer to the star than the section responsible for the main peaks. It is then difficult to conceive how the inner part of the same gap may be subject to a significant long term variability while the outer part remains comparatively stable. Furthermore, since in this framework the 
two main peaks are produced by two symmetrical outer gaps, it is difficult to explain why one is slightly more variable than its twin and why they produce different spectra (the possibility that the orientation of the pulsed emission highly depends on the energy would also contradict on statistical arguments the mere fact that we see the Vela and Crab pulsars in gamma rays). Finally, the spectral shape predicted by the outer gap model is not consistent with that of the two interpulse spectra. An alternative explanation is that active sites, other than the outer gaps created along the last open field lines, exist within the magnetosphere.

According to Cheng et al. (1986a), the main peaks come from two symmetrical gaps, one situated over each pole. In contrast, Morini (1983) and Smith (1986) propose a geometrical scheme where the two main gamma ray pulses are produced in the two sets of terminal field lines above the same pole and close to the light cylinder. This scheme assumes that the photons are emitted along the field lines and that the dipole is orthogonal to the spin axis. As in the other models, no correction could be introduced to account for the influence of the particle flow on the geometry of the lines. Morini uses this geometry to show that the gamma rays from the second peak may be contaminated by some polar cap radiation (both are seen at the same phase), whereas the first pulse is due entirely to radiation from the outer-magnetosphere. Assuming that relativistic particles produce synchro-Compton emission near the light cylinder (for the first peak and part of the second) and gamma rays near the polar cap by inverse Compton scattering of the thermal X-rays from the hot surface (for the rest of the second peak), Morini calculates spectra for these peaks which are consistent with the COS-B results. However, as the majority of the second peak gamma rays come from the polar cap component they should practically disappear below $50 \mathrm{MeV}$. This is not observed in the soft gamma ray lightcurve and therefore the mechanisms responsible for the high energy emission or their relative contributions require revision.

This scheme has been refined significantly by Smith (1986). By following this purely geometrical approach some of the new aspects of the pulsar behaviour may be discussed. As already indicated, the difference in the spectra between the two gamma ray peaks may come from the phase reconstruction. Only those photons created near the leading terminal field lines close to the light cylinder contribute to the first peak. On the other hand, "outer" photons from the trailing side may combine with photons from the same field lines deeper in the magnetosphere and also with photons from polar cap region to produce the second peak. Furtermore, the geometrical pattern indicates that the optical pulses are produced in the open magnetosphere above the pole, at a distance which is about half the light cylinder radius, and that the gamma ray interpulses originate along the same lines at a slightly higher altitude. The mixing in interpulse- 2 of photons created well inside the magnetosphere and near the polar cap could again explain the spectral differences with interpulse- 1 .

Assuming that the gamma ray interpulses do oridinate in the open magnetosphere, the spectral shape of interpulse- 1 has been compared with the predictions of various polar gap models (Daughterty and Harding, 1982; Heyvaerts and Signore, 1981; Ayasli, 1981). The lack of agreement between the predicted and measured shapes renders no clues to identify the mechanism responsible for the interpulse-1 radiation. Using the grid of spectra presented by Massaro and Salvati (1979) for various pulsar parameters, a good fit is obtained in the case of an initial Lorentz factor of $310^{7}$ for the primary particles and a low surface magnetic field value of $310^{11} \mathrm{G}$. However, the energy output from their model is not consistent with the COS-B observations of the interpulse.

The recent study by Caraveo et al. (1987) of possible polarisation of the Vela gamma ray emission using COS-B data also suggests that the peak and interpulse emissions originate in different parts of the magnetosphere. Their result, if confirmed, indicates a high degree of linear polarisation for the gamma rays coming from the entire interpulse, while the effect is much weaker for the second peak and not observed in the first peak. In the scheme proposed by Smith, a highly polarised gamma ray component deep in the open magnetosphere, created from synchrotron radiation or surviving selective absorption processes (Caraveo et al., 1987), would be consistent with the present observations.

The long-term variability of the gamma ray lightcurve reported in Sect. 5 also implies different source locations for the highly variable interpulse emissions and for the relatively stable main peaks. It is unlikely that the physical conditions may be variable on a time-scale from weeks to months, or stable, in regions along the same field lines. In the model presented by Smith, the various evolutions observed for the different gamma ray components would signify that the region sensitive to the variability is the open magnetosphere in the polar region, while the conditions along the last open field lines in the outer magnetosphere would be more steady. This situation may be tested with precise data on the evolution of the visual magnitude of the Vela pulsar. Alternatively, the inferred stability of the phenomena related to the outer gaps agrees with the steadiness of the Crab pulsar emission at all wavelengths. The synchronisation of its pulses from the optical range to gamma rays indicates that they are all emittted close to the light cylinder along the outer gap (Smith, 1986). For Vela, the details of the radio pulse profile and polarisation involve a source located close to the star polar cap (Krishnamohan and Downs, 1983). Therefore, the variability observed in radio supports the idea that the conditions in the inner magnetosphere may change in the long-term. The same conclusion is reached when considering that the largest contribution to the variability in the gamma ray domain comes from the low energy part, provided that in the cascade of photons the softer are produced deeper in the magnetosphere.

The qualitative discussion of the new aspects of the pulsed gamma ray emission in the geometrical model proposed by Smith suggests that the origin of the interpulses exists above one of the poles, deep in the magnetosphere or at medium altitudes, while the source of the peaks lies close to the co-rotating magnetosphere and to the light cylinder. However, further study is required into the possible co-existence of "polar" and "outer" gaps, and into the mechanisms which can explain the observed spectra. The model must also explain the non-existence of second pole radiation except in the radio range, the greater stability of the "outer" gaps over the "polar" gaps, and the different evolutions of the gamma ray and radio emissions. Finally, any model which tries to describe the Vela pulsar must at the same time explain the basic similarity of the Crab and Vela lightcurves at high energies and the marked differences in their overall behaviour.

\section{Conclusions}

The identification of the different components in the Vela gamma ray lightcurve supports the idea that several source regions exist 
in different parts of the pulsar magnetosphere. The significant contrast observed in the characteristics of the peak and interpulse emissions suggests that there may be at least two types of sources in the magnetosphere according to their location. The different spectral characteristics of the components indicate that different generating processes exist in each source. The origin of the variability observed in the low energy part of the gamma ray emission on time-scales from weeks to months must still be explained. In particular, with the present data, it is not possible to absolutely associate this phenomenon with the occurrence of giant glitches. Future satellite experiments, such as Gamma-1, Sigma and GRO, have lost their last gamma ray calibration candle, but will undoubtedly provide important information on the spectral characteristics of the various phase components and on their evolution. For the present, we have gained important information on the fundamental processes which govern the magnetosphere of a young pulsar.

Acknowledgements. We are grateful to Drs R. Buccheri and B. Sacco for the communication of the Vela timing parameters before the COS-B database release and to Dr. P. Thaddeus for the use of the Columbia CO survey. We thank Drs R. Buccheri and F. Lebrun for useful discussions. J. Clear acknowledges receipt of a research fellowship from the European Space Agency. The Laboratory for Space Research Leiden is supported by NWO, the Netherlands Organisation for the Advancement of Scientific Research.

\section{References}

Albats, P., Frye, G.M., Jr., Thomson, G.B., Hopper, V.D., Mace, O.B., Thomas, J.A., Staib, J.A.: 1974, Nature 251, 400

Ayasli, S.: 1981, Astrophys. J. 249, 698

Bennett, K., Bignami, G.F., Boella, G., Buccheri, R., Hermsen, W., Kanbach, G., Lichti, G.G., Masnou, J.L., Mayer-Hasselwander, H.A., Paul, J.A., Scarsi, L., Swanenburg, B.N., Taylor, B.G., Wills, R.D.: 1977, Astron. Astrophys. 61, 279

Bhat, P.N., Gupta, S.K., Ramana Murthy, P.V., Sreekantan, B.V., Tonwar, S.C., Viswanath, P.R.: 1980, Astron. Astrophys. 81, L3

Bhat, P.N., Gupta, S.K., Ramana Murthy, P.V., Sreekantan, B.V., Tonwar, S.C., Viswanath, P.R.: 1987, Astron. Astrophys. 178, 242

Bloemen, J.B.G.M., Strong, A.W., Blitz, L., Cohen, R.S., Dame, T.M., Grabelsky, D.A., Hermsen, W., Lebrun, F., MayerHasselwander, H.A., Thaddeus, P.: 1986, Astron. Astrophys. 154, 25

Buccheri, R., Caraveo, P.A., D’Amico, N., Hermsen, W., Kanbach, G., Lichti, G.G., Masnou, J.L., Wills, R.D., Manchester, R.N., Newton, L.N.: 1978, Astron. Astrophys. 69, 141

Buccheri, R., Bennett, K., Bignami, G.F., Bloemen, J.B.G.M., Boriakoff, V., Caraveo, P.A., Hermsen, W., Kanbach, G., Manchester, R.N., Masnou, J.L., Mayer-Hasselwander, H.A., Ozel, M.E., Paul, J.A., Sacco, B., Scarsi, L., Strong, A.W.: 1983, Astron. Astrophys. 128, 245

Buccheri, R., Di Gesu, V., Maccarone, M.C., Sacco, B.: 1987, Proc. 20th Int. Cosmic Ray Conf., Moscow, U.S.S.R., 1, 70

Caraveo, P.A., Bignami, G.F., Mitrofanov, I., Vacanti, G.: 1987, Astrophys. J. 327, 203

Cheng, K.S., Ho, C., Ruderman, M.: 1986a, Astrophys. J. 300, 500

Cheng, K.S., Ho, C., Ruderman, M.: 1986b, Astrophys. J. 300, 522

Clear, J., Bennett, K., Buccheri, R., Grenier, I.A., Hermsen, W.,
Mayer-Hasselwander, H.A., Sacco, B.: 1987, Astron. Astrophys. 174, 85

Cruise, A.M., Newton, A.C.: 1973, Nature Phys. Sci. 244, 121

Culhane, J.L., Cruise, A.M., Rapley, C.G., Hawkins, F.J.: 1976, Astrophys. J. Letters 190, L9

Dame, T.M., Ungerechts, H., Cohen, R.S., DeGeus, E.J., Grenier, I.A., May, J. Murphy, D.C., Nyman, L.-A., Thaddeus, P.: 1987, Astrophys. J. 322, 706

Daugherty, J.K., Harding, A.K.: 1982, Astrophys. J. 252, 337

Downs, G.S.: 1981 Astrophys. J. 249, 687

Downs, G.S., Manchester, R.N., Newton, L.M.: 1978, IAU Circ. 3274

Eadie, W.T., Drijard, D., James, F.E., Roos, M., Sadoulet, B.: 1977, Statistical Methods in Experimental Physics, North Holland, Amsterdam

Goldreich, P., Julian, W.H.: 1969, Astrophys. J. 157, 869

Gorenstein, M.V., Shapiro, I.I., Rogers, A.E.E., Cohen, N.L.: 1981, I AU Circ. 3644

Grindlay, J.E., Helmken, H.F., Hanbury-Brown, R., Davis, J., Allen, L.R.: 1975, Astrophys. J. 201, 82

Gupta, S.K., Ramana Murthy, P.V., Sreekantan, B.V., Tonwar, S.C., Viswanath, P.R.: 1982, Workshop on Very High Energy Gamma-ray Astronomy, Ootacamund, India, eds. Ramana Murthy and Weekes, 157

Harnden, F.R. jr, Johnson, W.N. III, Haymes, R.C.: 1972, Astrophys. J. Letters 172, L91

Harnden, F.R. jr, Grant, P.D., Seward, F.D., Kahn, S.M.: 1985, Astrophys. J. 299, 828

Harnden, F.R. jr, Gorenstein, P.: 1973, Nature 241, 107

Hermsen, W.: 1980, Ph.D. thesis, Univ. Leiden

Heyvaerts, J., Signore, M.: 1981, Astron. Astrophys. 96, 36

Kanbach, G., Bennett, K., Bignami, G.F., Buccheri, R., Caraveo, P., D’Amico, N., Hermsen, W., Lichti, G.G., Masnou, J.L., Mayer-Hasselwander, H.A., Paul, J.A., Sacco, B., Swanenburg, B.N., Wills, R.D.: 1980, Astron. Astrophys. 90, 163

Kellogg, E., Tananbaum, H., Harnden, F.R., jr, Gursky, H., Giacconi, R., Grindlay, J.: 1973, Astrophys. J. 183, 935

Knight, F.K.: 1982, Astrophys. J. 260, 538

Knight, F.K., Matteson, J.L., Peterson, L.E., Rothschild, R.E.: 1982, Astrophys. J. 260, 553

Krishnamohan, S., Downs, G.S.: 1983, Astrophys. J. 265, 372

Large, M.I., Vaughan, A.E., Mills, B.Y.: 1968, Nature 220, 340

Lichti, G.G., Buccheri, R., Caraveo, P., Gerardi, G., Hermsen, W., Kanbach, G., Masnou, J.L., Mayer-Hasselwander, H.A., Paul, J.A., Swanenburg, B.N., Wills, R.D.: 1980, Non-Solar Gamma Rays, Adv. Space Expl. Vol. 7 eds. R. Cowsik, R.D. Wills, Pergamon, Oxford, p. 49

Manchester, R.N., Goss, W.M., Hamilton, P.A.: 1976, Nature 259, 291

Manchester, R.N., Wallace, P.T., Peterson, B.A., Elliott, K.H.: 1980, Monthly Notices Roy. Astron. Soc. 190, 9P

Massaro, E., Salvati, M.: 1979, Astron. Astrophys. 71, 51

McAdam, W.B.: 1981, Proc. Astron. Soc. Australia 4, 219

Moore, W.E., Agrawal, P.C., Garmire, G.: 1974, Astrophys. J. Letters 189, L117

Morini, M.: 1983, Monthly Notices Roy. Astron. Soc. 202, 495

Mayer-Hasselwander, H.A.: 1985, Explanatory Supplement to the COS-B Final Database

Peterson, B.A., Murdin, P.G., Wallace, P.T., Manchester, R.N., Penny, A.J., Jorden, A., King, D., Hartley, K.F.: 1978, Nature 276, 475 
Pravdo, S.H., Becker, R.H., Boldt, E.A., Holt, S.S., Rothschild, R.E., Serlemitsos, P.J., Swank, J.H.: 1976, Astrophys. J. Letters 208, L67

Rappaport, S., Bradt, H., Doxsey, R., Levine, A., Spada, G.: 1974, Nature 251, 471

Ricker, G.R., Gerassimenko, M., McClintock, J.E., Ryckman, S.G., Lewin, W.H.G.: 1973, Astrophys. J. Letters 186, L111

Rickett, B.J., Seiradakis, J.H.: 1982, Astrophys. J. 256, 612

Salvati, M.: 1986, Astron. Astrophys. 154, 379

Scarsi, L., Bennett, K., Bignami, G.F., Boella, G., Buccheri, R., Hermsen, W., Koch, L., Mayer-Hasselwander, H.A., Paul, J.A., Pfeffermann, E., Stiglitz, R., Swanenburg, B.N., Taylor, B.G., Wills, R.D.: 1977, Proc. 12th ESLAB Symposium, Frascati, ESA SP-124, p. 3

Shapiro, S.L., Teukolsky, S.A.: 1983, Black Holes, White Dwarfs and Neutron Stars; The Physics of Compact Objects (John Wiley and Sons, New York)

Smith, F.G.: 1986, Monthly Notices Roy. Astron. Soc. 219, 729

Strong, A.W., Bloemen, J.B.G.M., Lebrun, F., Hermsen, W.,
Mayer-Hasselwander, H.A., Buccheri, R.: 1987, Astron. Astrophys. Suppl. 67, 283

Strong, A.W., Bloemen, J.B.G.M., Dame, T.M., Grenier, I.A., Hermsen, W., Lebrun, F., Nyman, L.-A., Pollock, A.M.T., Thaddeus, P.: 1987, Proc. 20th Int. Cosmic Ray Conf., Moscow, U.S.S.R. 1, 125

Thompson, D.J., Fichtel, C.E., Kniffen, D.A., Ogelman, H.B.: 1977, Astrophys. J. Letters 214, L17.

Tümer, O.T., Dayton, B., Long, J., O’Neill, T., Zych, A., White, R.S.: 1984, Nature 310, 214

Wallace, P.T., Peterson, B.A., Murdin, P.G., Danziger, I.J., Manchester, R.N., Lyne, A.G., Goss, W.M., Smith, F.G., Disney, M.J., Hartley, K.F., Jones, D.H.P., Wellgate, G.W.: 1977, Nature 266, 692

Wills, R.D., Bennett, K., Bignami, G.F., Buccheri, R., Caraveo, P.A., Hermsen, W., Kanbach, G., Masnou, J.L., Mayer-Hasselwander, H.A., Paul, J.A., Sacco, B.: 1981, Proc. 17th Int. Cosmic Ray Conf., Paris, France, XG1.2-8

Zimmermann, H.V.: 1980, Astron. Astrophys. 88, 309. 